\title{
1 C. elegans episodic swimming is driven by multifractal kinetics
}

\section{Author Affiliation}

3 Yusaku Ikeda ${ }^{1,2, \uparrow}$, Peter Jurica ${ }^{1, \uparrow}$, Hiroshi Kimura ${ }^{2}$, Hiroaki Takagi ${ }^{3}$, Struzik Zbigniew ${ }^{4,5,6}$, Ken Kiyono ${ }^{7}$,

4 Yukinobu Arata ${ }^{1, *}$, and Yasushi Sako ${ }^{1}$

5 1. Cellular Informatics Laboratory, RIKEN, 2-1 Hirosawa, Wako 351-0198, Saitama, Japan

6 2. Department of Mechanical Engineering, School of Engineering, Tokai University, 4-1-1 Kitakaname, 7 Hiratsuka, Kanagawa 259-1292, Japan

8 3. Department of Physics, Nara Medical University, 840 Shijocho, Kashihara, Nara 634-8521, Japan.

9 4. Graduate School of Education, University of Tokyo, 7-3-1 Hongo, Bunkyo-ku, Tokyo 113-0033, Japan

0 5. Advanced Center for Computing and Communication, RIKEN, 2-1 Hirosawa, Wako 351-0198, Saitama, Japan

6. Faculty of Physics, University of Warsaw, Pasteur 5, 02-093 Warsaw, Poland

3 7. Graduate School of Engineering Science, Osaka University, 1-3 Machikaneyama-cho, Toyonaka, Osaka 5604 8531, Japan.

$5 \dagger$ these authors contributed equally

6 *To whom correspondence may be addressed. Email arata@riken.jp

\section{Author contributions}

8 Conceptualization: Yukinobu Arata and Hiroaki Takagi

9 Formal analysis: Peter Jurica and Yukinobu Arata,

0 Funding acquisition: Yukinobu Arata and Yasushi Sako

1 Investigation: Yusaku Ikeda, Yukinobu Arata, and Peter Jurica

2 Methodology: Yusaku Ikeda and Hiroshi Kimura

3 Writing: original draft: Yukinobu Arata

4 Writing - review \& editing: Hiroaki Takagi, Struzik Zbigniew, Ken Kiyono, and Yasushi Sako

6 The authors declare no conflict of interest.

7 This article contains supporting information online at

8 This open access article is distributed under Creative Commons Attribution License (CC BY).

\section{$0 \quad$ Data deposition}


1 The C. elegans swimming activity time series and movie data reported in this paper have been deposited in

2 Systems Science of Biological Dynamics (SSBD) database ${ }^{1}$, http://ssbd.qbic.riken.jp/set/20190401/

\section{Keywords}

4 microfluidic device, cGMP-dependent kinase (PKG), scale-free, power law, multifractal, temporal correlation

\section{Abstract}

7 Fractal scaling is a common property of temporal change in various modes of animal behavior. The molecular 8 mechanisms of fractal scaling in animal behaviors remain largely unexplored. The nematode C. elegans alternates

9 between swimming and resting states in a liquid solution. Here, we report that C. elegans episodic swimming is 0 characterized by scale-free kinetics with long-range temporal correlation and local temporal clusterization, which 1 is characterized as multifractal kinetics. Residence times in actively-moving and inactive states were distributed 2 in a power law-based scale-free manner. Multifractal analysis showed that temporal correlation and temporal 3 clusterization were distinct between the actively-moving state and the inactive state. These results indicate that $C$. 4 elegans episodic swimming is driven by transition between two behavioral states, in which each of two transition 5 kinetics follows distinct multifractal kinetics. We found that a conserved behavioral modulator, cyclic GMP 6 dependent kinase $(\mathrm{PKG})$ may regulate the multifractal kinetics underlying an animal behavior. Our combinatorial 7 analysis approach involving molecular genetics and kinetics provides a platform for the molecular dissection of 8 the fractal nature of physiological and behavioral phenomena.

\section{$0 \quad$ Abbreviations}

$1 \quad$ cGMP-dependent kinase $(\mathrm{PKG})$ 


\section{Introduction}

3 Animal behaviors are organized over a broad range of time scales, ranging from seconds to years, including

4 expansive timescales over lifespan phases, such as infant, juvenile, adult, and elderly phases. Among the

5 temporally organized animal behaviors are rhythmic behaviors characterized by their frequencies. Molecular

6 regulators e.g. for daily rhythmic and oscillatory changes of animal behaviors i.e. for circadian rhythm have been

7 identified and are shown to be regulated by a feedback control ${ }^{2,3}$. Contrastingly, many arrhythmic changes of

8 behavioral and physiological activities in a great variety of animal species, including humans are reported to show

9 self-similar and scale-free structures, which is an indicative for fractal scaling ${ }^{4-6}$. Fractal geometry provides a

0 mathematical framework for characterizing scale-free and self-similar patterns ${ }^{4-6}$. Fractal temporal patterns of

1 behavior and physiology have been reported in the locomotion of birds, mosquito larvae, and flies ${ }^{7-9}$, crawling

2 of cultured C. elegans worms ${ }^{10}$, clicking sounds produced by feeding sea horses ${ }^{11}$, and swimming of zooplankton

3 12,13. In humans, temporal fractal patterns have been observed in wrist movements during habitual sleep/wake

4 cycles $^{14}$, gait ${ }^{15,16}$, heartbeats ${ }^{17,18}$, and brain activity ${ }^{19,20}$. Altered fractal patterns of activity have been associated

5 with pathological conditions and aging ${ }^{14-16,18,21,22}$, indicating that the fractal patterning in biological activity is a

6 critical measure for characterizing physiological status. Thus, fractal scaling is a clue to study molecular and

7 physiological basis of arrhythmic and complex animal behaviors, and there may be molecular and physiological

8 mechanisms that adhere to the basic principles of fractal geometry. However, the molecular and physiological

9 mechanisms remain unknown.

Owing to its simple anatomy and the availability of a range of genetic tools, C. elegans is a powerful

1 model organism for the study of the molecular bases of behavior. In solution, C. elegans worms alternate between

2 swimming and resting states on a minute to hour time scale ${ }^{23,24}$. In the swimming state, they alternate between

3 continuously beating their bodies and resting with or without food ${ }^{23-25}$. In the resting state, they maintain a

4 characteristic sharply-bent posture ${ }^{23}$. Episodic swimming is conserved in nematodes cultured in a liquid solution

$5 \quad{ }^{23}$. On a solid agar plate, C. elegans also move in an episodic manner, wherein they crawl actively and persistently 
6 in one direction or crawl slowly and stay within a small area, behavioral states called roaming and 7 dwelling/quiescence, respectively ${ }^{24,26,27}$. Individual Drosophila flies ${ }^{9}$ and Leptothorax allardycei worker ants ${ }^{28}$ 8 also alternate between an actively-moving state and a resting state in an episodic manner. Thus, episodic behavior 9 is a conserved presentation in invertebrates that is thought to be adaptive for supporting food exploration, energy 0 conservation, and reproductive success ${ }^{9,23,24,29}$.

2 PKG is a behavior modulating enzyme conserved across invertebrates and vertebrates ${ }^{30,31}$. C. elegans egl-4/pkg 3 mutants roam continuously on a solid agar plate and swim when in a solution with less frequent resting than is 4 exhibited by wild-type animals 23,25-27. Because egl-4/pkg-dependent regulation is found in both medium 5 conditions ${ }^{23,25-27}$, C. elegans episodic motions in both conditions are thought to be regulated by the same 6 molecular/physiological mechanism ${ }^{24}$. In Drosophila, the foraging/pkg homolog of egl-4 had been discovered 7 from natural behavioral polymorphisms wherein flies tend to travel long distances (rover) or be sedentary (sitter) $8 \quad 32,33$. The expression level of foraging/pkg differs between rover flies and sitter flies and Drosophila traveling 9 behavior can be switched by genetic manipulation of foraging/pkg ${ }^{34}$. Foraging behaviors in social insects0 including those exhibited by honey bees (Apis mellifera) and ants (Pheidole pallidula) that are determined by 1 developmental stage ${ }^{35}$ and social caste ${ }^{36}$, respectively - are also associated with PKG expression and activity. 2 Thus, PKG is considered to be a conserved modulator of animal behavior ${ }^{30,31}$.

To study scaling of behavior across a broad range of time scales, it is necessary to obtain behavioral 4 activity time series of individual animals for an extended period of time at a high temporal resolution. In this 5 study, we recorded the swimming behavior of 108 individual C. elegans at a semi-video rate for a week-long 6 period by individually culturing the worms in a newly developed microfluidic device. The obtained time series 7 data encompassing $10^{7}$ time points was submitted to multifractal analysis. We found that C. elegans episodic 8 swimming is a scale-free process and the transition between actively-moving and inactive states is driven in 9 multifractal kinetic. Additionally, we examined the sensitivity of the multifractal kinetics of the behavior to 
0 modulation by PKG. A discussion of potential molecular and physiological mechanisms underlying

1 multifractality in behavior is provided on the basis of previously reported mathematical and physical models.

\section{Results}

\section{C. elegans episodic swimming is a multi-time scale process}

5 Swimming of multiple adult $C$. elegans individuals was monitored in a newly developed microfluidic device,

6 called WormFlo, which is equipped with an array of 108 disc-shaped chambers for culturing individual C. elegans

17 (Fig. 1A and B). C. elegans individuals were maintained alone in chambers under controlled chemical, 8 temperature, and light intensity conditions (Fig. S2, Fig. S3, and Methods). We recorded swimming at $10^{7}$ time

9 points with a $50-\mathrm{ms}$ interval $(138 \mathrm{~h} \approx 5.8 \mathrm{~d})$, and quantified swimming activity with a pixel counting method (Fig.

0 2A, Fig. S4, Movie S1, Methods, and Supplemental Information). In the absence of an energy source, the animals'

1 swimming activity diminished gradually over time (Fig. 2B and Movie S2). The swimming activity in WormFlo

2 chambers decayed with kinetics similar to that seen in animals cultured without an energy source in substantially

3 larger $(2 \times$ diameter, $23 \times$ volume) 96 -well plate wells (Movie S3); and the activity was sustained in animals

4 cultured in WormFlo with an energy source (glucose and cholesterol) (Fig. S5A and Movie S4). Therefore, the

5 activity decay observed can be attributed primarily to a physiological response to long-term cultivation without

6 an energy source rather than to physical damage or spatial restriction.

Comparing the activity decay kinetics over the 6-d observation period among individuals led us to

8 define three empirical activity classes: High, long-term high activity; Middle, intermediate-term high activity;

9 and Low, short-term high activity (Fig. 2B). The early and late culturing periods were defined as Pre-starved and

0 Starved time regimes, each of which has high and low swimming activities (for quantitative criteria, see Fig. 2B

1 legend, and Methods). Consistent with previous studies, we observed episodic swimming bouts on minute to hour

2 time scales (Fig. 2C; 8-min scale and 80-min scale), and a characteristic kinked posture during the resting state

3 in the Pre-starved regime (data not shown) ${ }^{23,24}$. 
Interestingly, we observed a swimming bout cluster on a 1 day-scale that could be divided in several

5 clusters in a magnified view of about $1 \mathrm{~h}$ (Fig. 2C, compare the red bracketed region in the 14-h scale vs. red

6 arrows in the 80-min scale). This nested temporal structure was observed repeatedly over a series of

7 magnifications from a 1-h scale to a 10-min scale (Fig. 2C, red region in 80-min scale vs. red arrows in 8-min

8 scale), and between the 1-min and 1-s scales (Fig. 2C, red bracket region in 8-min scale vs. red arrows in 50-s

9 and 5-s scales). In the 1-s scale, animals alternated between bending their bodies and beating their bodies for

0 swimming (Movie S1). Series of beating motions were interrupted with intermittent short resting periods, referred

1 as to "posing" (Movie S1). During posing, they were transiently motionless in a bent posture (Movie S1, see

2 legend). The temporal resolution at which continuous shape could be detected in H265 codec-compressed movies

3 was $0.25 \mathrm{~s}$ (Fig. S6 and Supplemental Information), which was sufficient to detect subsecond "posing" on a 1-s

4 scale. The temporally nested structure of C. elegans episodic motion was observed on time scales of a

5 magnification range of about 1,000 times. Thus, C. elegans episodic swimming has multi-timescale dynamics

6 with a self-similar temporal structure.

\section{A scale-free property in $C$. elegans episodic swimming}

9 Continuous swimming ceased and resumed suddenly (Fig. 2C). Consistently, we observed a bimodal probability

0 distribution of activity strength (Fig. 3A), indicating that $C$. elegans episodic swimming can be characterized by

1 a two-state transition model between actively-moving and inactive states. To reveal the kinetics of the state

2 transition, we studied the statistical distribution of residence times in active and inactive states. Active and inactive

3 states were defined as periods above or below an activity threshold, which was the value in the bimodal

4 distribution valley (Fig. 3A) of swimming activity time series (red horizontal line in Fig. 2C, 5-s scale). Residence

5 time in the two states were obtained alternately from swimming activity time series; eventually, we obtained

6 round series data for active and inactive states (Fig. 3B and C). Residence times in the two states were distributed 
7 along a broad temporal range, ranging from subsecond to 10 -s orders for the active state and ranging from

8 subsecond to 100 -s orders for the inactive state (Fig. 3B and C).

The probability distributions of residence times in active and inactive states were linear lines in log-log

0 plots or power law distributions (Fig. 3D-F, and Table 1, fit parameter), indicating that the state residence times

1 lack a specific time scale and thus can be described as exhibiting a scale-free property. Comparing the power law

2 exponents between the two states in each activity class in Pre-starved and Starved time regime, we find that the

3 power law relationship slopes are significantly shallower for the inactive state than for the active state (a "shallow

4 slope" power law relationship indicates a relatively even appearance of longer and shorter residence times, Fig.

5 3D-F, Table 1 fit parameters and $\mathrm{p}$ values in Table 1). These results indicate that the mechanisms that regulate

6 the transition from the active to the inactive state and from the inactive to the active state have distinct scale-free

7 kinetics.

\section{Multifractal analysis of numerically-generated round series data}

0 Long and short bursts of state residence times in the round series data were clustered rather than random (Fig. 3B

1 and C). We employed multifractal detrended fluctuation analysis (MF-DFA, Methods) to study the temporal

2 structure of the residence-time round series data ${ }^{37,38}$. First, time series of white, pink, and Brown noises were

3 numerically-generated by R software tools for fractional Gaussian noise generation ${ }^{39,40}$, and were subjected to

4 MF-DFA. White noise time series is completely uncorrelated, whereas pink and Brown noise time series have

5 long-range correlation. Noise components of cumulatively summed original time series $[f(v, s)$ in Eq. 1$]$ was

6 estimated in MF-DFA by removing local trends in each scale, $s$, by linear fitting to various time scales (Methods).

7 As the temporal correlation in the time series became longer, the noise component (i.e. deviation from the trend

8 of cumulative sums of deviations from the average) became smoother (Fig. 4A; upper three series). The calculated

9 average noise component $[F(q, s)$, Eq. 1] was plotted with temporal resolution for observation in $s$. In $F(q, s)$

$0 \quad$ versus $s$ plots on a $\log -\log$ scale, $F(q, s)$ increased linearly with increasing scale $s$ (Fig. 4A; left column of lower 
1 three graphs), as is indicative of fractal scaling. Additionally, the slope of $F(q, s)$ versus $s$ plot on a $\log -\log$ scale

2 increased with an increasing length of temporal correlation of the original time series (Fig. 4A; left column of

3 lower three graphs). The $F(q, s)$ versus $s$ plot gave rise to a multifractal spectrum (Methods and Supplemental

4 Information) showing a relationship between $q$-order (local) Hurst exponent (Hölder spectrum $H(q)$ ) vs. $q$-order

5 singularity dimension (singularity spectrum $D(q))^{37,38}$.

The singularity dimension is a fractal dimension that represents time series sparsity; a $D(q)<1$

represents sparsely distributed local structures of the entire time series, and a $D(q)$ approaching 1 representing

broadening involvement of the time series. Thereby, the Hurst exponent at $D(q)<1$ represents a Hurst exponent

9 of local structures of time series, which is the local Hurst (Hölder) exponent, whereas the Hurst exponent at $D(q)$

$0=1$ represents the Hurst exponent of the entire time series, which we call the global Hurst exponent $\left(H_{p e a k}\right)$. The

1 Global Hurst exponent $H_{\text {peak }}$ corresponds approximately to the conventional Hurst exponent, which is linked

2 theoretically with the scaling exponent of power spectrum density $\left[\beta,\left(S(f) \sim f^{-\beta}\right)\right]$, a measure of time series

3 autocorrelation $[\beta=2 H+1,(H>0)]$ (Kiyono, 2015). Therefore, the global Hurst exponent $H_{\text {peak }}$ can be

4 interpreted as an index of behavioral memory in activity time series of behavior. The $H_{\text {peak }}$ values of the

5 cumulative sum of these noise time series were estimated to be approximately $0.5,1$, and 1.5 (Fig. $4 \mathrm{~A}$; right

6 column of lower three graphs), consistent with theoretical Hurst exponents of cumulative sums of these noise

7 time series.

Next, multifractal time series were numerically-generated by multiplicative cascading process ${ }^{41,42}$, and were subjected to MF-DFA. The multifractal time series were highly clusterized, such that the variety of amplitudes and durations of temporal clusters became richer with greater multifractality (Fig. 4B; upper three series). Because of the scale-free and self-similar properties of multifractal time series, such temporal clusters are 2 distributed in multiple temporal resolutions. As the variety of amplitudes and durations of temporal clusters 3 increased, the $F(q, s)$ versus $s$ plot slope in a $\log$-log scale varied widely with the exponentiation factor $q$ (Fig. 4 4B; left column of lower three graphs), which enhances or suppresses the noise component from local trends at 
5 scale $s[f(v, s)$ in Eq. 1, and Methods]. The $q$-dependent change in slope in a $\log$-log scale $F(q, s)$ versus $s$ plot

6 is indicative of multifractal scaling. In accordance with this $q$-dependent variation, the width of the multifractal

7 spectrum (width) widens significantly without a substantial change in $H_{\text {peak }}$ (Fig. 4B; right column of lower three

8 graphs). A wide multifractal spectrum indicates that there are locally clustered structures with various local Hurst

9 exponents sparsely distributed throughout the time series. Therefore, multifractal spectrum width in animal

0 activity time series is an index of behavioral complexity. Note that global Hurst exponent $H_{\text {peak }}$ and multifractal

1 spectrum width are independently changeable in a scale-free time series with a given power law exponent. See

2 the Supplemental Information for detailed descriptions of numerical generation for noise time series and

3 multifractal analysis, and for an introduction into fractal scaling.

5 Episodic swimming is driven by two-state transition with two distinct multifractal kinetics

6 Global trend in the round series data of active- and inactive-state residence times became gradually shorter and 17 longer, respectively, after transition from the Pre-starved to the Starved time regime ( 15,000 rounds; Fig. 3B

8 and C). The noise components of cumulative sums of the deviations from average residence times appeared to

9 differ qualitatively between active and inactive states; that of active states was smoothly curved, whereas that of

0 inactive states was locally straighter in the Pre-starved regime (Fig. 4C).), suggesting that noise properties of

1 active and inactive states may differ. In the $\log$-log scale $F(q, s)$ versus $s$ plot, $F(q, s)$ in active and inactive states

2 increased almost linearly with $s$ and the slope varied with $q$ (Fig. 4C; left column of lower two graphs), and the

3 multifractal spectra of active state and inactive states in the Pre-starved time regimes were widely distributed (Fig.

$44 \mathrm{C}$; right column of lower two graphs), indicating that the round series of active and inactive states had a 5 multifractal nature.

The Global Hurst exponents $H_{\text {peak }}$ in multifractal spectra of active and inactive states were located

7 around $H(q)=1$, indicating that the state residence times have a non-trivial long-range temporal correlation, as is 8 seen in pink noise time series. As shown in Figure 5A-C, before and after starvation in all activity classes, we 
9 observed greater $H_{\text {peak }}$ values for the inactive state (average, 1.38) than for the active state (average, 0.85$)$ as well

0 as greater width values in the inactive state (average, 1.80) than in the active state (average, 0.64 ), all $\mathrm{p}<0.05$ ).

1 Hence, residence-time round series revealed distinct multifractal properties between active and inactive states,

2 with a longer behavioral memory and greater behavioral complexity for the inactive state than for the active state.

3 We called kinetics that generate scale-free residence times with temporal correlation and temporal clusterization

4 as multifractal kinetics. Accordingly, we were able to explain C. elegans episodic swimming with a two-state

5 transition model in which opposite transitions between actively-moving and inactive states are driven by distinct

6 multifractal kinetics (Fig. 6A).

The power law exponent showed a more pronounced alteration between in Pre-starved and Starved time

8 regimes in residence-time round series for the inactive state (average, 22.4\%) than for the active state (average,

$9 \quad 7.0 \%$ ). The change in the power law exponent was only statistically significant for the inactive state ( $p$ values and

0 fit parameters in Table 1). MF-DFA showed that the global Hurst exponent $H_{\text {peak }}$ was not altered significantly by

1 the transition to the Starved time regime in either the active state (average Pre-starved $H_{p e a k}, 0.86$; average Starved

$\left.2 H_{\text {peak }}, 0.84\right)$ or the inactive state (average Pre-starved $H_{\text {peak }}, 1.34$; average Starved $\left.H_{p e a k}, 1.42\right)\left(H_{p e a k}\right.$ values, and

$3 \mathrm{p}$ values in Table 2, all $\mathrm{p}>0.05)$. However, the multifractal spectrum width was altered after starvation, becoming

4 narrower $(-17.1 \%)$ in the active state and wider $(+31.6 \%)$ in the inactive state (width values, and $\mathrm{p}$ values in Table

52 , all $\mathrm{p}<0.05$ except active state in Low class, see Figure 5 legend). These results indicate that food signaling or

6 metabolic state regulates behavior by modulating multifractal kinetics in response to starvation. That is, in

7 response to food starvation, the animals do not simply decrease swimming activity, but show a selective 8 modulation of multifractal kinetics.

\section{$0 \quad$ PKG may regulate the multifractal kinetics of $C$. elegans episodic swimming}

1 For molecular dissection of the multifractal kinetics C. elegans episodic swimming, we studied the egl-4(n479) 2 mutant, in which the temperature sensitive $n 479$ allele of egl-4 is associated with a defect in conventional episodic 
3 swimming characterized by lengthened continuous swimming periods and a reduced frequency of resting ${ }^{23,25}$.

4 We confirmed that egl-4(n479) mutants had reduced frequencies of a persistent inactive state in our quantitative

5 activity time series (Fig. S5B). The egl-4(n479) mutants cultured in WormFlo without an energy source were too

6 transparent for body detection in the later portion of the 6-d culture period. This transparency was likely caused

7 by the mutants' unusually early lipid consumption due to their continuous swimming and lack of long resting;

8 lipids in worm bodies scatter illuminating light enabling imaging as a (relatively) dark object as shown in Figure

9 2A. Thus high-contrast images of the first tenth of the 6-d culturing period were used for analysis of the Pre-

$0 \quad$ starved time regime of egl-4 mutants.

In egl-4 mutants, the power law relationships were maintained in active and inactive states, but the

2 power law exponent was preferentially changed in the inactive state $(+21.0 \%)$ compared to that in active state

3 (+6.9\%) (Fig. 3G and D). Power law exponents differed significantly between wild-type worms and egl-4 mutants

4 only in the inactive state ( $\mathrm{p}$-values and fit parameters in Table 1), indicating that the mutant has altered residence

5 times across time scales rather than a defect that is specific to a particular time scale. MF-DFA showed that the

6 shape of the multifractal spectrum in egl-4 mutants was largely altered in the inactive state (Figs. 4D and 5D).

7 Comparing the global Hurst exponent between egl-4 mutants and High-activity class wild-type worms, we found

8 that active-state $H_{\text {peak }}$ was similar across the groups (difference, $1.1 \%$ ), whereas inactive-state $H_{\text {peak }}$ differed

9 significantly between the groups (difference, 18.7\%). Meanwhile, spectrum width in egl-4 mutants was reduced

0 significantly relative to that obtained for the High activity wild-type group in both active (-20.9\%) and inactive

$1 \quad(-14.1 \%)$ states ( $H_{\text {peak }}$ and width values, p values in Table 2$)$. Thus, egl-4 mutants showed a defect in both temporal

2 memory and temporal clusterization in the inactive state, but showed a defect in only temporal clusterization in

3 the active state. These results are consistent with the possibility that EGL-4/PKG may regulate the multifractal

4 kinetics of behavior, and, more specifically, suggest that EGL-4/PKG may regulate the active- and inactive-state

5 kinetics of C. elegans episodic swimming differently. 


\section{Discussion}

\section{C. elegans episodic swimming is driven by a multifractal transition cycle}

9 Experimental measurements of temporal changes in biological system variables of interest and the statistical

0 analysis of those data can provide information about the hidden operating principles of that system ${ }^{43}$. C. elegans

1 episodic swimming was characterized conventionally by the average residence time of active- and inactive-states

2 23-25,44. Here, we applied fractal analysis to study the molecular and genetic mechanisms regulating animal

3 behavior over a 6-d period recorded at a subsecond temporal resolution, showing that episodic swimming is a

4 scale-free process across a 1000-fold range of time scales (Fig. 2C). Because the experimentally-measured

5 average residence times often fail to be determined by values that greatly exceeded the average times, the scale-

6 free process is characterized by a power law exponent in the relationship between appearance frequency and

7 residence time (Supplemental Information). Our multifractal analysis showed long-range memory and local

8 clusterized structures of episodic swimming residence times characterized by a multifractal process. Hence, we

9 referred to the swimming as "multifractal episodic swimming" (Fig. 6B).

A scale-free distribution of actively-moving and inactive residence times has been reported for episodic

1 Drosophila behavior 9; modeling suggested that the scale-free nature of these residence times contributes to

2 maximizing the food exploitation area while optimizing food intake time. Previous analyses have shown long-

3 range temporal correlations in scale-free properties of the behaviors of many species, including fractal (but not

4 multifractal) analysis of $C$. elegans crawling 7,8,10-13. The fractal nature of C. elegans crawling in agar (Alves et

5 al., 2017) is consistent with our long-range memory and clusterization finding. In this study, we extended previous

6 findings by combining a two-state transition model with multifractal analysis. Employing multifractal analysis,

7 we found that scale-free actively-moving and inactive states had a long-range memory and complex local

8 temporal structures. Our analysis indicated that $C$. elegans episodic swimming is characterized by a two-state

9 transition between actively-moving and inactive states, wherein the two transitions are driven by distinct

0 multifractal kinetics. That is, the active-to-inactive transition is driven by a narrow multifractal kinetics (short 
1 long-range temporal correlation and low complexity), whereas the inactive-to-active transition is driven by a wide

2 multifractal kinetics (extended long-range temporal correlation and relatively high complexity) (Fig. 6A).

Multifractal episodic motion of C. elegans in solid and liquid environments may be an adaptation to

4 food environments. The colonies of bacteria that $C$. elegans worms feed on grow in a fractal shape ${ }^{45}$. In $C$.

5 elegans, the actively-moving state is likely to enable food foraging, whereas the inactive state is likely associated

6 with food intake, egg-laying, or resting to save energy or satiety ${ }^{24,29-31,46,47}$. Temporal correlation in the inactive

7 state gives rise to a series of long and short periods for food intake that may follow the fractally-shaped bacterial

8 colony, whereas temporal correlation in active state round series gives rise to a series of long- and short-distance

9 foraging bouts that may follow the interbranch distances of a fractally-shaped bacterial colony. Thus, the scale-

0 free and temporally structured residence times for food foraging and intake may be adaptive to the fractal shape

1 of bacterial colonies. C. elegans survival strategies are altered by food availability. Under starvation conditions,

2 C. elegans saves their energy for long-distance foraging and instead spend more effort for balancing resting and

3 food intake at a local area, e.g. through reuptake of their excrements. Food-dependent modulation of multifractal

4 kinetics of behaviors may improve food intake efficiency and reproductive success in natural environments. This

5 possibility should be tested in a modeling study.

Our quantitative studies showed that egl-4 mutants exhibited different alterations of multifractal

17 kinetics in the active versus the inactive state (Fig. 6C). This result suggests that EGL-4 may regulate the 8 multifractal kinetics of animal behaviors. However, egl-4 mutants' defects in behavioral memory and behavioral 9 complexity were smaller in magnitude than the differences between the active and inactive states in wild-type $C$. 0 elegans, and also smaller than the index that characterizes qualitative differences of noise properties among white, 1 pink, and Brown noises. Differences between active and inactive states in wild-type C. elegans were comparable 2 to or greater than the index (compare $H_{p e a k}$ in Table 2: $H_{\text {peak }}$ of white, pink, and Brown noises differ by 0.5 ). 3 Therefore, multifractal kinetics in egl-4 mutants were within the range of kinetics qualitatively same as those in 4 wild-type. In addition, these defects may be caused by a behavioral variation of the egl-4 mutant strain rather than 
5 by loss of function of egl-4/pkg per se. It is necessary to apply other fractal analyses and further molecular/genetic

6 studies to examine these possibilities.

$8 \quad$ PKG-modulated multifractal transition may be widely applicable to multi-time scale behaviors

9 Multifractal transition cycle and its PKG-dependent modulation may be shared among many invertebrates. In 0 short-term (sub-minute) observations, Drosophila ${ }^{9}$ and Leptothorax allardycei worker ants ${ }^{28}$ exhibit bimodal

1 behavioral mode switching ${ }^{9,28}$ similar to that in C. elegans. Notably, Drosophila residence times in actively2 moving and inactive states in a short-term observation period were distributed in a scale-free manner ${ }^{9}$. 3 Interestingly, a bimodal behavioral choice between long-distance moving foragers and dwellers has been shown 4 to be regulated by PKG in C. elegans ${ }^{26,27,48}$, Drosophila ${ }^{32}$, bees ${ }^{35}$, and ants ${ }^{36,49}$. Both C. elegans mutant ${ }^{26,27}$ 5 and Drosophila polymorphism ${ }^{32,50}$ local dwellers spend more time on local food intake/resting. Local dwellers 6 conduct brood care in bee hives ${ }^{35}$ and ant nest defense ${ }^{36,49}$. Forager and dweller behavioral phenotypes are 7 switched developmentally in bees, but maintained through the lifespan in the ant caste system. In C. elegans ${ }^{26,27,48}$ 8 and ants ${ }^{36}$, long-distance foraging is associated with low PKG activity or expression, whereas local dwelling is 9 associated with high PKG expression. Conversely, long-distance foraging (or local dwelling) is associated with 0 high (low) activity or expression of PKG in flies ${ }^{32}$ and bees ${ }^{35}$. The time scale difference of PKG-active periods 1 between ants and honey bees, and the reversed function of PKG for long-distance foraging or local dwelling 2 between nematodes/ants and flies/bees may reflect a heterochronic evolutionary change in the developmental 3 control of PKG expression ${ }^{35}$, and an evolutionary adaptation of the PKG signaling system in molecular drivers 4 of behavior, respectively. Despite evolutionary changes in time scale and the functional role of PKG, these results 5 indicate that appearance frequency for actively-moving and inactive states is modulated by PKG. Although the 6 role of PKG in the episodic motions of Drosophila and ants has not been studied directly, the aforementioned 7 findings suggest that at least episodic motions in Drosophila and ants may be regulated by multifractal kinetics 8 and its PKG-dependent modulation. 
0 be involved in human heartbeat physiology. Electrocardiogram time series ${ }^{51-53}$ of cardiac muscle depolarization 1 and repolarization are characterized by multifractality. The inter-beat (RR) interval is the period between peaks

2 of R waves, which reflect ventricular depolarization. The intra-beat (QT) interval is the period from the peak of

3 Q wave to the end of $\mathrm{T}$ wave, which corresponds to the depolarization in the left side of the intraventricular

4 septum and the repolarization of ventricular muscles, respectively. Round series of RR and QT intervals are

5 characterized by a multifractal structure ${ }^{54}$. Interestingly, the shape of the multifractal spectrum for RR intervals

6 differs from that for QT intervals, raising the possibility that QT and RR intervals may be regulated by distinct

7 multifractal kinetics. PKG has been shown to regulate the heartbeat in mice and flies ${ }^{55-58}$. PKG-knockout mice

856 or cardio-myocyte specific PKG-knockout mice ${ }^{58}$ show hypertension. Although the mechanism is still in

9 dispute ${ }^{57}$, the defects in these knockout mice indicate that PKG is involved in the relaxation phase of the cardiac

0 cycle. Thus, the heartbeat can be regulated by a multifractal transition cycle and is subject to PKG-dependent

1 modulation, similar to that we documented for C. elegans episodic swimming. Thus, PKG-modulated multifractal

2 transition cycles may occur in various animal species, including humans, and organs.

$4 \quad$ Molecular and system-level mechanisms for multifractal episodic swimming

5 Based on previously reported models designed to recapitulate scale-free and multifractal time series, we 6 developed hypotheses for molecular and system-level mechanisms underlying the multifractal nature of animal 7 behaviors, and discuss how PKG may be involved in the mechanism. First, the intermittent bursts of a single 8 neuron can be reproduced in chaos dynamical systems ${ }^{59-65}$; owing to their non-Gaussian fluctuation with 9 intermittency, such systems may be associated with multifractality in animal behaviors. Chaotic dynamics 0 reproduced in these models are generated by interactions between fast inflows and slow outflows of ions to/from 1 a neuron. Thus, multifractal nature in behavior might be attributable to chaotic biochemical reactions in a single 2 neuron. Second, when a system is in a certain state, called the criticality, local interactions among system 
3 components related to order and noise for randomization of the order reach a critical balance and, eventually, self-

4 similar dynamics emerge spontaneously in the system ${ }^{66,67}$; scale-free dynamics due to the criticality can be 5 associated with multifractality in animal behaviors ${ }^{68,69}$. Experimentally observed scale-free neural activity in

6 intact brains was recapitulated in self-organized criticality-based models that assumed global interaction of

7 neuronal signals in a hierarchically structured neural network representative of brain structures in C. elegans and

8 humans ${ }^{70,71}$. Therefore, multifractality in animal behaviors may be attributed to criticality in the hierarchal

9 structure of brains. Third, although implications of molecular and physiological mechanisms in the multifractal

0 nature of animal behaviors have not been reported, the multiplicative cascade model is a simple model that

1 generates multifractal time series (Fig. 4B, Supplemental Information) ${ }^{41,42,72,73}$. Generally, multiplicative cascade

2 models generate multifractal time series via a series of multiplications of log-normal random noise in a cascade

3 manner, enhancing time series variance greatly as it progresses (see Supplemental Information). It has been shown

4 that waiting time distributions of neuronal action potentials exhibit a log-normal distribution consequent to the

5 nonlinearity of reaction systems in neurons ${ }^{74-76}$, whereas hierarchal multiplication may correspond to a cascading

6 signal relay in the brain. Thus, multifractal nature in behaviors may be attributed to both non-linear reactions

7 within neurons and hierarchal structures of neural networks. To reveal molecular- and system-level mechanisms

8 of C. elegans multifractal episodic swimming, it will be critical to identify the operating principles that are

9 functioning in multifractal episodic swimming in C. elegans. With regard to PKG, selective ectopic expression

0 of PKG in R3 and R4d ring neurons is sufficient to restore behavioral defects in Drosophila with pkg/foraging

1 alleles ${ }^{77,78}$. Additionally, differential PKG expression was identified in a set of five specific neurons between

2 different castes of ants ${ }^{36}$. In C. elegans, PKG functions in a limited number of neurons ${ }^{25}$. PKG has been shown

3 to regulate synaptic vesicle cycling, $\mathrm{Ca}^{2+}$ influx via G-protein signaling, and axon guidance in certain neuron

4 types ${ }^{77,79-82}$. The endogenous and ectopic expression of PKG in specific neurons and functional analysis of PKG

5 suggest that PKG modulates multifractal kinetics of animal behaviors in single neurons or small numbers of

6 neurons. In this study, our experiments revealed a basic kinetic mechanism of C. elegans multifractal episodic 
7 swimming. The findings are applicable to diverse fields of interest, including human heartbeat physiology. Due

8 to the wide variety of molecular and genetic tools available for C. elegans research, our observation and analysis

9 approach may be used to help reveal conserved mechanisms underlying the multifractal nature of animal

0 physiology and behavior.

2 Methods

3 C. elegans strains used and their maintenance

4 The Bristol N2 strain was used as wild-type C. elegans. N2 and egl-4(n479) mutant animals were maintained on 5 agar plates with E. coli OP50 strain at $15^{\circ} \mathrm{C}{ }^{83}$.

\section{Design, fabrication, and characterization of microfluidic device}

8 To maximize the number of animals monitored in the recording area, our device employs a two-vertical-

9 compartment structure with the array of culture chambers located over a flow path, whose boundary was

0 partitioned by a porous membrane (Whatman 111115, Nuclepore Hydrophilic Membrane, 10- $\mu \mathrm{m}$ pores, GE

1 Healthcare, USA) (Fig. 1A and B). The upper polydimethylsiloxane (PDMS) chip consisted of a 108-chamber

2 array (1 worm/chamber) with buffer-inlet and exchange solution-outlet ports in the chambers. The lower

3 PDMS chip consisted of a snake-shaped microchannel for supplying liquid buffer to the culture chambers. PDMS

4 chips were fabricated by conventional replica molding with the SU-8 epoxy-based photoresist ${ }^{84,85}$. Animal-

5 loading ports $(100 \mu \mathrm{m}$ wide) on the top of each of chamber in the upper PDMS chip were made with a Zing 16

6 laser (Epilog Laser, Japan). Before culturing and buffer exchanges, the loading ports were sealed with a PDMS

17 sheet. Each culture chamber (2-mm diameter and 0.3-mm height) in the upper PDMS chip was approximately 2

8 fold-longer and 3 fold-thicker than the $\sim 1$-mm-long and $\sim 0.1$-mm-wide C. elegans body (Fig. 1C). The serpentine

9 buffer supply microchannel in the lower PDMS chip had a 2.2-mm diameter, covering each upper-chip culture 
0 chamber. The three parts (upper and lower PDMS chips, and the microporous membrane) were assembled by

1 covalent bonding with an aminosilane coupling agent and oxygen plasma treatment ${ }^{86}$.

3 Culturing $C$. elegans in a microfluidic device

4 Animals at the young adult stage were collected from an agar plate. After an agar plate-culture period ( $2 \mathrm{~d}$ at $5 \quad 24{ }^{\circ} \mathrm{C}$ for wild-type and $3 \mathrm{~d}$ at $15^{\circ} \mathrm{C}$ for egl-4 mutants), the animals were collected manually in room-temperature

6 M9 buffer and introduced into the microfluidic device by manual pipetting via chamber loading ports (Fig, 1C).

$7 \quad$ With previous devices, individual C. elegans were held in a clump structure and released into the chamber by 8 applying deforming high pressure ${ }^{87-89}$. We instead used manual pipetting to avoid applying mechanical stress 9 during loading. To maintain a constant chemical environment in the culture chambers, M9 buffer (with or without $0 \quad 1 \mathrm{~g} / \mathrm{L}$ glucose and $5 \mathrm{mg} / \mathrm{L}$ cholesterol) was perfused continuously with a peristatic pump (Fig. 1B and D) at a flow 1 rate at $5 \mathrm{ml} / \mathrm{h}$. Flow rate was measured by monitoring weight changes in the water discharged from the outlet 2 (data not shown). Buffer exchange in chambers with the flow passing through the porous membrane was 3 confirmed in experiments with a fluorescent solution (Fig. S2). Air bubbles in the buffer supply tube were 4 removed with polytetrafluoroethylene membrane in an Omnifit bubble trap (006BT, Diba Industries Inc., USA) 5 (Fig. 1D).

\section{Observation of $C$. elegans swimming}

8 C. elegans behaviors were recorded at 20 frames/s through a macroscope with an apochromat objective lens $(1 \times)$ 9 (Z16 APO, Leica, Germany) and a CCD camera $(1940 \times 1460$ pixels, 2.8 Megapixel) with a USB3.0 connection 0 (MD028MU-SY, Ximea, Germany). The camera was controlled by Micromanager (https://micro-manager.org). 1 Movies were compressed (H265 codec in FFmpeg) every 10,000 frames (500 s) into mp4 files. C. elegans are 2 sensitive to temperature change of $4{ }^{\circ} \mathrm{C}{ }^{90}$ and highly sensitive to blue-ultraviolet light, but not to green/yellow 3 light $(>545 \mathrm{~nm})^{91,92}$. Temperature of culture chambers on WormFlo were maintained by submerging WormFlo 
4 in M9 buffer in a 15-cm-diameter glass dish, whose temperature was maintained by temperature-controlled water

5 supplied from a high-precision water bath (HAAKE, Germany) (Fig. 1D and Fig. S3A and B). A temperature

6 logger (TC-08, Pico Technology, UK) confirmed that the temperature of the M9 buffer in the glass dish was

7 maintained within $\pm 0.5^{\circ} \mathrm{C}$ during the 6-day recording period (Fig. S3C). The recording system was covered by a

8 light shield to prevent illumination changes from light fluctuations related to daily lab activities (Fig. 1D). Blue

9 light of illumination light from a halogen lamp was filtered out with a 0.5 -mm-thick orange acryl plate (Fig. 1D

0 and S3A); spectrophotometry (USB400, Ocean Optics, USA) confirmed that wavelengths $<500$ nm were filtered

1 out (Fig. S3D). Standard deviation of light intensity change at the culture chambers during observation period

2 was kept within $10 \%$ of the average (Fig. S3E). This culturing and recording system allowed us to monitor

3 individually cultured C. elegans in an environment with minimized chemical, light, and temperature perturbations.

\section{Quantification of $C$. elegans swimming activity}

6 We measured $C$. elegans swimming activity by counting the number of pixels with an intensity over a certain

7 threshold in a matrix obtained from the difference between intensities obtained at $\mathrm{t}$ and $\mathrm{t}+1$ (we referred to it as

8 image difference) using the Open CV module in Python2.7. When an animal moves at a frame interval, the dark

9 pixels detecting its body in image[t] become brighter in image $[\mathrm{t}+1]$, yielding an increased image difference $(=$

$0 \quad$ image $[\mathrm{t}+1]-$ image[t]) (Fig. 2A). For pixel counting, the image difference matrix values were in the range of -

1256 to +256 at $1940 \times 1460$ pixels. The pixels with intensity differences greater than 12 value within this range

2 thus exhibit an image difference and are counted as "active" pixels (note that the pixel intensity threshold of 12

3 is different from the activity threshold (12 pixels/frame) to define residence time in active- and inactive states

4 (Fig. 3A)). The pixel intensity threshold of 12 was determined empirically so that actual movements of animals

5 are detected efficiently while avoiding artefact-based false hits due to thermal noise in a pixel on the camera

6 sensor. Based on pixel counts, the size of the area the animal moved through during each time frame (50 ms) was

7 measured (Fig. 2A). Image and data processing, including compensation for artifactual activity in the time series 
8 are described in the Supplemental Information (Figs. S4 and S6). To classify swimming activity, we measured

9 average activities during the early half of the recording period (day 0 to day 3; abbreviated "AA0-3") and the

0 ratio of AA0-3 to average activities during the latter half of the recording period (day 3 to day 6 ; abbreviated 1 “AA3-6"). Animals with high AA0-3 and a low AA0-3 to AA3-6 ratio were classified as High activity. Animals 2 with high AA0-3 and a high AA0-3 to AA3-6 ratio were classified as Middle activity. Animals with low AA0-3 3 and a low AA0-3 to AA3-6 ratio were classified as Low activity. The threshold of high AA0-3 was set empirically 4 at 0.75 and the threshold of a high ratio was set to 0.01 . Note that 0.75 threshold values are low due to the 5 bursty/sparse nature of C. elegans swimming activity.

\section{Data analysis}

8 Active-state residence time was defined as the period after the start of the activity burst to the end of the burst; 9 inactive-state residence time was defined as the period starting immediately after the end of activity burst to the 0 next round of activity burst in the activity time series. Alternating-state round series data were obtained by 1 thresholding time series of swimming activity at 12 pixels/frame, which corresponded to the valley of a bimodal 2 activity distribution (Fig. 3A).

MF-DFA was performed in Python software ${ }^{93}$ using

$$
F(q, s)=\frac{1}{2 N_{s}}\left\{\sum_{v=1}^{2 N_{s}}\left[f^{2}(v, s)\right]^{q / 2}\right\}^{1 / q}
$$

where $f(v, s)$ is the noise component or fluctuations from the local trend of cumulative sums of the deviation of

residence time from the average residence times in the $v^{\text {th }}$ segment at the temporal resolution for observation $(s)$.

MF-DFA was derived from detrended fluctuation analysis (DFA) ${ }^{94}$; the equation used in DFA corresponds to the equation used in MF-DFA when $q=2$ in Eq. 1). In operation 1, the entire cumulative sum series was segmented into Ns segments at scale $s$, and the local trend in each $v^{\text {th }}$ segment at scale $s$ was determined by piecewise fitting

with a linear function. In operation 2, the amplitude of fluctuations from the local linear trend at each segment

1 was enhanced (or suppressed) to a large (or small) amplitude of $f(v, s)$ by exponentiating with positive (or 
2 negative) $q$-values; $q$ value-exponentiated $f(v, s)$ was summed over all the $v^{\text {th }}$ segments. In operation 3 . the 3 operations 1 and 2 above were performed bidirectionally, forward and backward, on the round series (in total $2 \times$

$4 \quad N_{s}$ segments) to obtain $F(q, s) . F(q, s)$ versus $s$ plots were $\log$-log plotted, and each $q$-value was fit with a linear 5 function to obtain a local Hurst (Hölder) exponent. Finally, multifractal spectrum [ $q$-order (local) Hurst exponent, 6 or Hölder spectrum $H(q)$ vs. $q$-order singularity dimension, or singularity spectrum $D(q)$ ] was obtained from $7 F(q, s)$ versus $s$ at each $q$-value by Legendre transformation. Linear fitting to data in a log-log plot minimizes 8 relative error between the fit function (y_fit) and the data (y_data), which is $\log \left(\mathrm{y}_{-}\right.$fit $/ \mathrm{y}_{-}$data) $\left(=\log \left(\mathrm{y} \_\right.\right.$fit $)-$ 9 log(y_data $))$. Linear fitting to data in a log-log plot avoids biased fitting due to errors being weighted by high$0 \quad$ value data points that happens when fitting is performed by minimizing absolute error (y_fit - y_data). Animals 1 whose chambers had long-term retained bubbles, and wild-type animals which were transparent at the final movie 2 frame were eliminated from the data analysis.

\section{Statistical analysis}

5 Student t-tests and chi-squared tests for independence were performed with scipy.stats.chi2_contingency and scipy.stats.ttest_ind, respectively 95 .

$8 \quad$ Funding

9 Y. Arata is supported by a research grant, Challenging Research (Pioneering), Grants-in-Aid for Scientific 0 Research, Ministry of Education, Culture, Sports, Science and Technology, Japan (18H05300).

\section{Acknowledgements}

3 We thank the Caenorhabditis Genetics Center (CGC) for C. elegans strains; the CGC is funded by NIH Office 4 of Research Infrastructure Programs (P40 OD010440). We are grateful to Yuki Shindo for providing valuable 5 help with Python programing, to Seiichi Uchida at Kyushu University for sharing computational power for 
i recording, and Hideitsu Hino at The Institute of Statistical Mathematics for advices for statistical tests. We are

7 grateful to RStudio Team (2018) for RStudio: Integrated Development for R. RStudio, Inc., Boston, MA URL 8 http://www.rstudio.com/.

\section{$0 \quad$ References}

11 Tohsato, Y., Ho, K. H., Kyoda, K. \& Onami, S. SSBD: a database of quantitative data of spatiotemporal 2 dynamics of biological phenomena. Bioinformatics 32, 3471-3479, doi:10.1093/bioinformatics/btw417 3 (2016).

42 Reddy, A. B. \& Rey, G. Metabolic and nontranscriptional circadian clocks: eukaryotes. Annu Rev Biochem

83, 165-189, doi:10.1146/annurev-biochem-060713-035623 (2014).

63 Mellor, J. The molecular basis of metabolic cycles and their relationship to circadian rhythms. Nat Struct Mol Biol 23, 1035-1044 (2016).

84 Iannaccone, P. M. \& Khokha, M. Fractal Geometry in Biological Systems: An Analytical Approach. (CRC Press, 1996).

5 Mandelbrot, B. B. The fractal geometry of nature. Updated and augm. edn, (W.H. Freeman, 1983).

16 Bunde, A. \& Havlin, S. Fractals in Science. (Springer-Verlag, 1994).

27 Kembro, J. M., Flesia, A. G., Gleiser, R. M., Perillo, M. A. \& Marin, R. H. Assessment of long-range correlation in animal behavior time series: The temporal pattern of locomotor activity of Japanese quail (Coturnix coturnix) and mosquito larva (Culex quinquefasciatus). Physica A 392, 6400-6413 (2013).

Guzman, D. A. et al. The fractal organization of ultradian rhythms in avian behavior. Sci Rep 7, 684, doi:10.1038/s41598-017-00743-2 (2017).

Cole, B. J. Fractal Time in Animal Behavior - the Movement Activity of Drosophila. Anim Behav 50, 1317-1324 (1995). 
910 Alves, L. G. A. et al. Long-range correlations and fractal dynamics in C. elegans: Changes with aging and

Stress. Phys Rev E 96 (2017).

111 Haris, K., Chakraborty, B., Menezes, A., Sreepada, R. A. \& Fernandes, W. A. Multifractal detrended fluctuation analysis to characterize phase couplings in seahorse (Hippocampus kuda) feeding clicks. $J$ Acoust Soc Am 136, 1972-1981, doi:10.1121/1.4895713 (2014).

Seuront L, M.C., B. \& J.R., S. in Handbook of Scaling Methods in Aquatic Ecology: Measurement, Analysis, Simulation (eds Laurent Seuront \& Peter G. Strutton) 333 (CRC Press, 2003).

Seuront, L., Schmitt, F. G., Brewer, M. C., Strickler, J. R. \& Souissi, S. From random walk to multifractal random walk in zooplankton swimming behavior. Zool Stud 43, 498-510 (2004).

Sci U S A 106, 2490-2494, doi:10.1073/pnas.0806087106 (2009).

Hausdorff, J. M. et al. Altered fractal dynamics of gait: reduced stride-interval correlations with aging and Huntington's disease. J Appl Physiol (1985) 82, 262-269, doi:10.1152/jappl.1997.82.1.262 (1997).

Hausdorff, J. M. Gait dynamics, fractals and falls: finding meaning in the stride-to-stride fluctuations of human walking. Hum Mov Sci 26, 555-589, doi:10.1016/j.humov.2007.05.003 (2007).

17 Kobayashi, M. \& Musha, T. 1/f fluctuation of heartbeat period. IEEE Trans Biomed Eng 29, 456-457, doi:10.1109/TBME.1982.324972 (1982).

18 Ivanov, P. C. et al. Scaling behaviour of heartbeat intervals obtained by wavelet-based time-series analysis. Nature 383, 323-327, doi:10.1038/383323a0 (1996).

19 Stam, C. J. \& de Bruin, E. A. Scale-free dynamics of global functional connectivity in the human brain. Hum Brain Mapp 22, 97-109, doi:10.1002/hbm.20016 (2004).

20 Linkenkaer-Hansen, K., Nikouline, V. V., Palva, J. M. \& Ilmoniemi, R. J. Long-range temporal correlations and scaling behavior in human brain oscillations. J Neurosci 21, 1370-1377 (2001). 
321 Struzik, Z. R., Hayano, J., Sakata, S., Kwak, S. \& Yamamoto, Y. 1/f scaling in heart rate requires antagonistic autonomic control. Phys Rev E Stat Nonlin Soft Matter Phys 70, 050901, doi:10.1103/PhysRevE.70.050901 (2004).

22 Goldberger, A. L. et al. Fractal dynamics in physiology: alterations with disease and aging. Proc Natl Acad Sci U S A 99 Suppl 1, 2466-2472, doi:10.1073/pnas.012579499 (2002).

Ghosh, R. \& Emmons, S. W. Episodic swimming behavior in the nematode C. elegans. J Exp Biol 211, 3703-3711, doi:10.1242/jeb.023606 (2008).

McCloskey, R. J., Fouad, A. D., Churgin, M. A. \& Fang-Yen, C. Food responsiveness regulates episodic behavioral states in Caenorhabditis elegans. J Neurophysiol 117, 1911-1934, doi:10.1152/jn.00555.2016 (2017).

Ghosh, R. \& Emmons, S. W. Calcineurin and protein kinase G regulate C. elegans behavioral quiescence during locomotion in liquid. BMC Genet 11, 7, doi:10.1186/1471-2156-11-7 (2010). by sensory perception and the EGL-4 cGMP-dependent protein kinase. Neuron 36, 1091-1102 (2002).

L'Etoile, N. D. et al. The cyclic GMP-dependent protein kinase EGL-4 regulates olfactory adaptation in

C. elegans. Neuron 36, 1079-1089 (2002).

28 Cole, B. J. Short-Term Activity Cycles in Ants - Generation of Periodicity by Worker Interaction. Am Nat 137, 244-259, doi:Doi 10.1086/285156 (1991).

29 McConnell, M. W. \& Fitzpatrick, M. J. 'Foraging' for a place to lay eggs: A genetic link between foraging behaviour and oviposition preferences. Plos One 12 (2017).

30 Sokolowski, M. B. Social interactions in "simple" model systems. Neuron 65, 780-794, doi:10.1016/j.neuron.2010.03.007 (2010). 
Osborne, K. A. et al. Natural behavior polymorphism due to a cGMP-dependent protein kinase of

Drosophila. Science 277, 834-836, doi:DOI 10.1126/science.277.5327.834 (1997).

34 Kaun, K. R. et al. Natural variation in food acquisition mediated via a Drosophila cGMP-dependent protein kinase. J Exp Biol 210, 3547-3558, doi:10.1242/jeb.006924 (2007). different time scales on behavior. Science 296, 741-744, doi:10.1126/science.1069911 (2002).

Lucas, C. \& Sokolowski, M. B. Molecular basis for changes in behavioral state in ant social behaviors. Proc Natl Acad Sci U S A 106, 6351-6356, doi:10.1073/pnas.0809463106 (2009).

Kantelhardt, J. W. et al. Multifractal detrended fluctuation analysis of nonstationary time series. Physica A 316, 87-114 (2002).

Ihlen, E. A. Introduction to multifractal detrended fluctuation analysis in matlab. Front Physiol 3, 141, doi:10.3389/fphys.2012.00141 (2012).

Beran, J., Whitcher, B. \& Maechler, M. longmemo; Statistics for Long-Memory Processes (Book Jan Beran), and Related Functionality, R package Version 1.1-1. (2018).

Ihaka, R. \& Gentleman, R. R: A Language for Data Analysis and Graphics. Journal of Computational and Graphical Statistics 5, 299-314, doi:10.2307/1390807 (1996).

Bacry, E., Delour, J. \& Muzy, J. R. Multifractal random walk. Phys Rev E 64 (2001).

42 Kiyono, K., Struzik, Z. R. \& Yamamoto, Y. Estimator of a non-Gaussian parameter in multiplicative lognormal models. Phys Rev E 76 (2007).

43 Arata, Y. \& Takagi, H. Quantitative Studies for Cell-Division Cycle Control. Frontiers in Physiology 10, doi:10.3389/fphys.2019.01022 (2019). 
044 Gonzales, D. L., Zhou, J., Fan, B. \& Robinson, J. T. A microfluidic-induced C. elegans sleep state. Nat

Commun 10, 5035, doi:10.1038/s41467-019-13008-5 (2019).

45 Matsuyama, T. \& Matsushita, M. Fractal morphogenesis by a bacterial cell population. Crit Rev Microbiol

19, 117-135, doi:10.3109/10408419309113526 (1993).

You, Y. J., Kim, J., Raizen, D. M. \& Avery, L. Insulin, cGMP, and TGF-beta signals regulate food intake and quiescence in C-elegans: A model for satiety. Cell Metab 7, 249-257 (2008).

Raizen, D. M. et al. Lethargus is a Caenorhabditis elegans sleep-like state. Nature 451, 569-U566 (2008).

Raizen, D. M., Cullison, K. M., Pack, A. I. \& Sundaram, M. V. A novel gain-of-function mutant of the cyclic GMP-dependent protein kinase egl-4 affects multiple physiological processes in Caenorhabditis elegans. Genetics 173, 177-187, doi:10.1534/genetics.106.057380 (2006).

Ingram, K. K., Oefner, P. \& Gordon, D. M. Task-specific expression of the foraging gene in harvester ants. Mol Ecol 14, 813-818, doi:10.1111/j.1365-294X.2005.02450.x (2005).

Osborne, K. A., de Belle, J. S. \& Sokolowski, M. B. Foraging behaviour in Drosophila larvae: mushroom body ablation. Chem Senses 26, 223-230, doi:10.1093/chemse/26.2.223 (2001). (Wolters Kluwer Health, 2016).

Dubin, D. Rapid Interpretation of EKGs. 6th edn, (Cover Pubulishing Co, 1998). doi:10.1016/j.cmpb.2012.02.014 (2012).

Lewis, M. J., Short, A. L. \& Suckling, J. Multifractal characterisation of electrocardiographic RR and QT time-series before and after progressive exercise. Comput Methods Programs Biomed 108, 176-185, Johnson, E., Sherry, T., Ringo, J. \& Dowse, H. Modulation of the cardiac pacemaker of Drosophila: cellular mechanisms. J Comp Physiol B 172, 227-236, doi:10.1007/s00360-001-0246-8 (2002). 
356 Pfeifer, A. et al. Defective smooth muscle regulation in cGMP kinase I-deficient mice. EMBO J 17, 30453051, doi:10.1093/emboj/17.11.3045 (1998).

557 Feil, R., Lohmann, S. M., de Jonge, H., Walter, U. \& Hofmann, F. Cyclic GMP-dependent protein kinases and the cardiovascular system: insights from genetically modified mice. Circ Res 93, 907-916, doi:10.1161/01.RES.0000100390.68771.CC (2003).

Wegener, J. W. et al. cGMP-dependent protein kinase I mediates the negative inotropic effect of cGMP in the murine myocardium. Circ Res 90, 18-20 (2002).

Fan, Y. S. \& Holden, A. V. Bifurcations, Burstings, Chaos and Crises in the Rose-Hindmarsh Model for

Neuronal-Activity. Chaos Soliton Fract 3, 439-449 (1993).

Gu, H. G. \& Xiao, W. W. Difference Between Intermittent Chaotic Bursting and Spiking of Neural Firing Patterns. Int J Bifurcat Chaos 24 (2014).

61 Izhikevich, E. M. Neural excitability, spiking and bursting. Int J Bifurcat Chaos 10, 1171-1266 (2000).

562 Chay, T. R. Chaos in a 3-Variable Model of an Excitable Cell. Physica D 16, 233-242 (1985).

663 Fan, Y. S. \& Chay, T. R. Generation of periodic and chaotic bursting in an excitable cell model. Biol

Cybern 71, 417-431 (1994).

64 Holden, A. V. \& Fan, Y. From Simple to Complex Oscillatory Behaviour via Intermittent Chaos in the

Rose-Hindmarsh Model For Neuronal Activity. Chaos, Solitons \& Fractals 2, 349-369, doi:https://doi.org/10.1016/0960-0779(92)90012-C (1992).

65 Canavier, C. C., Clark, J. W. \& Byrne, J. H. Routes to chaos in a model of a bursting neuron. Biophys J 57, 1245-1251, doi:10.1016/S0006-3495(90)82643-6 (1990).

Beggs, J. M. The criticality hypothesis: how local cortical networks might optimize information processing. Philos T R Soc A 366, 329-343 (2008). Lett 59, 381-384, doi:10.1103/PhysRevLett.59.381 (1987). 
768 Schertzer, D. \& Lovejoy, S. Multifractal Generation of Self-Organized Criticality. Ifip Trans A 41, 325$8 \quad 339(1994)$.

969 Tebaldi, C., De Menech, M. \& Stella, A. L. Multifractal scaling in the Bak-Tang-Wiesenfeld sandpile and edge events. Physical Review Letters 83, 3952-3955 (1999).

170 Rubinov, M., Sporns, O., Thivierge, J. P. \& Breakspear, M. Neurobiologically realistic determinants of self-organized criticality in networks of spiking neurons. PLoS Comput Biol 7, e1002038, doi:10.1371/journal.pcbi.1002038 (2011).

471 Moretti, P. \& Munoz, M. A. Griffiths phases and the stretching of criticality in brain networks. Nat Commun 4, 2521, doi:10.1038/ncomms3521 (2013).

672 Arneodo, A., Manneville, S., Muzy, J. F. \& Roux, S. G. Revealing a lognormal cascading process in turbulent velocity statistics with wavelet analysis. Philos T R Soc A 357, 2415-2438 (1999).

873 Meneveau, C. \& Sreenivasan, K. R. The Multifractal Nature of Turbulent Energy-Dissipation. J Fluid Mech 224, 429-484 (1991).

74 Petersen, P. C. \& Berg, R. W. Lognormal firing rate distribution reveals prominent fluctuation-driven regime in spinal motor networks. Elife 5, doi:10.7554/eLife.18805 (2016).

275 Buzsaki, G. \& Mizuseki, K. The log-dynamic brain: how skewed distributions affect network operations. Nat Rev Neurosci 15, 264-278 (2014).

476 Roxin, A., Brunel, N., Hansel, D., Mongillo, G. \& van Vreeswijk, C. On the distribution of firing rates in networks of cortical neurons. $J$ Neurosci 31, 16217-16226, doi:10.1523/JNEUROSCI.1677-11.2011 (2011).

77 Peng, Q. et al. cGMP-Dependent Protein Kinase Encoded by foraging Regulates Motor Axon Guidance in Drosophila by Suppressing Lola Function. J Neurosci 36, 4635-4646, doi:10.1523/JNEUROSCI.3726$15.2016(2016)$. 
078 Kuntz, S., Poeck, B., Sokolowski, M. B. \& Strauss, R. The visual orientation memory of Drosophila requires Foraging (PKG) upstream of Ignorant (RSK2) in ring neurons of the central complex. Learn Mem 19, 337-340, doi:10.1101/lm.026369.112 (2012). retrograde mechanism for exoendocytic coupling of synaptic vesicles. Neuron 74, 517-529, doi:10.1016/j.neuron.2012.03.028 (2012).

80 Wang, X. \& Robinson, P. J. Cyclic GMP-dependent protein kinase and cellular signaling in the nervous system. J Neurochem 68, 443-456, doi:10.1046/j.1471-4159.1997.68020443.x (1997).

81 Dason, J. S., Allen, A. M., Vasquez, O. E. \& Sokolowski, M. B. Distinct functions of a cGMP-dependent protein kinase in nerve terminal growth and synaptic vesicle cycling. $J$ Cell Sci 132, doi:10.1242/jcs.227165 (2019).

82 Krzyzanowski, M. C. et al. The C. elegans cGMP-dependent protein kinase EGL-4 regulates nociceptive behavioral sensitivity. PLoS Genet 9, e1003619, doi:10.1371/journal.pgen.1003619 (2013).

83 Brenner, S. The genetics of Caenorhabditis elegans. Genetics 77, 71-94 (1974).

Lorenz, H. et al. High-aspect-ratio, ultrathick, negative-tone near-UV photoresist and its applications for MEMS. Sensor Actuat a-Phys 64, 33-39 (1998).

Hosokawa, K., Fujii, T. \& Endo, I. Handling of picoliter liquid samples in a poly(dimethylsiloxane)-based microfluidic device. Anal Chem 71, 4781-4785 (1999).

Kimura, H., Yamamoto, T., Sakai, H., Sakai, Y. \& Fujii, T. An integrated microfluidic system for longterm perfusion culture and on-line monitoring of intestinal tissue models. Lab on a Chip 8, 741-746 (2008).

Chung, K. et al. Microfluidic chamber arrays for whole-organism behavior-based chemical screening. Lab Chip 11, 3689-3697, doi:10.1039/c1lc20400a (2011). of C. elegans reproductive aging. Lab Chip 15, 524-531, doi:10.1039/c4lc01028k (2015). 
489 Hulme, S. E. et al. Lifespan-on-a-chip: microfluidic chambers for performing lifelong observation of C. 5 elegans. Lab Chip 10, 589-597, doi:10.1039/b919265d (2010).

690 Simonetta, S. H., Migliori, M. L., Romanowski, A. \& Golombek, D. A. Timing of locomotor activity circadian rhythms in Caenorhabditis elegans. PLoS One 4, e7571, doi:10.1371/journal.pone.0007571 (2009).

991 Edwards, S. L. et al. A novel molecular solution for ultraviolet light detection in Caenorhabditis elegans. 0 PLoS Biol 6, e198, doi:10.1371/journal.pbio.0060198 (2008).

192 Ward, A., Liu, J., Feng, Z. \& Xu, X. Z. Light-sensitive neurons and channels mediate phototaxis in $C$. 2 elegans. Nat Neurosci 11, 916-922, doi:10.1038/nn.2155 (2008).

393 Jurica, P. Multifractal analysis for all. Frontiers in Physiology 6, doi:UNSP 27

$4 \quad 10.3389 /$ fphys.2015.00027 (2015).

594 Peng, C. K., Havlin, S., Stanley, H. E. \& Goldberger, A. L. Quantification of scaling exponents and crossover phenomena in nonstationary heartbeat time series. Chaos 5, 82-87, doi:10.1063/1.166141 (1995).

895 Jones, E., Oliphant, T., Peterson, P. \& others. SciPy: Open Source Scientific Tools for Python [Online; accessed 2019-04-09], <"http://www.scipy.org/"> (2001). 


\section{Figure legends}

\section{Figure 1 Culturing and recording system for individual $C$. elegans animals}

4 (A and B) The WormFlo apparatus has a vertically two-compartment structure, wherein an array of 108 culture 5 chambers and loading ports of the upper PDMS chip are partitioned from the buffer flow path located in the lower PDMS chip by a porous membrane (details in the Methods). M9 buffer was supplied from the inlet and withdrawn

7 from the outlet. (C) The diameter and height of each culture chamber in the upper PDMS chip were $2 \mathrm{~mm}$ and

$80.3 \mathrm{~mm}$, approximately 2-fold longer and 3-fold thicker than the $\sim 1$-mm-long and $\sim 0.1$-mm wide C. elegans

9 body, respectively. Animals are introduced in each chamber via a 0.1-mm-wide loading port on the roof of a disc-

0 shaped culture chamber, which was closed with a thin PDMS sheet before M9 buffer was perfused. (D) C. elegans

1 animals cultured in the WormFlo are monitored by a macroscope with a CCD camera. Buffer perfusion is driven

2 by a peristatic pump. To avoid loss of water due to evaporation, the WormFlo was submerged in a 15 -cm-diameter

3 glass dish (depth, $5.4 \mathrm{~cm}$ ) filled with M9 buffer. The temperature of the M9-filled 15-cm glass dish was

4 maintained by circulating temperature-controlled water in a water flow path in the attached aluminum block under

5 the 15-cm glass dish (Fig. S3A). Animals cultured in the microfluidic device are illuminated by blue-light filtered

6 light from a halogen lamp in a light shielding box that buffers against light intensity changes due to daily lab 7 activity.

9 Figure 2 C. elegans episodic swimming exhibited a multi-time scale kinetics.

0 Swimming activity of animals cultured in individual chambers was quantified by a pixel counting method 1 (Methods). (A) Chambers in WormFlo are shown with row-column indexes (left figure). Active pixels (intensity 2 difference $>12$ in the range of -256 to 256 ) between time point [t] and time point [ $t+1]$ are shown in red pixels in 3 the upper right image and in yellow pixels in the lower right image, respectively. The animal in chamber id 54 4 moved while the animal in culture chamber id 66 did not move from time [t] to [t+1]. (B) Active pixel numbers 
5 are shown on the y-axis as an index of swimming activity with culture time is shown on the x-axis. Six-day

6 temporal activity decay patterns were classified into High, Middle, and Low activity classes by two criteria;

7 average activities during the early half of the recording period (first 3 days) and the ratio of average activity in

8 the early half to that in the late half of the recording period (see Methods). Pre-starved regime (red area) and

9 Starved regime (blue area) were defined for each activity class. For the High activity class, the Pre-starved and

0 Starved regimes were defined by early $50 \%$ period from the start of recording through the early half of the

1 recording period and the remainder of the period. For the Middle activity class, Pre-starved regime and Starved

2 regime were defined by the period from the start of recording to early $20 \%$ period of the recording period and the

3 remainder. For the Low activity class, the Pre-starved and Starved regimes were defined by the period from the

4 start of recording to early $10 \%$ of the recording period and the remainder. (C) Swimming activities of a

5 representative animal in various time scales; full recording time scale ( $10^{7}$ timepoints) at the top. Each of the

6 lower panels are a 10× magnification of the first tenth of its upper panel (red area). Activity threshold at 12

7 pixels/frame is shown by a red horizontal line in the 5-s scale. Animal activities at $6 \mathrm{~d}-, 1 \mathrm{~d}-, 1 \mathrm{~h}-, 10 \mathrm{~min}-, 1 \mathrm{~min}-$,

8 and 1 s-scales are shown.

$0 \quad$ Figure 3 A power law distribution of active/inactive state residence times in $C$. elegans 1 episodic swimming

2 (A) Appearance frequency of number of active pixels in a representative animal for a single image difference

3 frame. Bimodal distribution of active pixels in normalized probability density (NPD) was separated at 10 20

4 pixels/frame. The active state threshold was $\geq 12$ active pixels for round series data analysis (details in Methods).

5 (B and C) Round series of residence times in the active (B) and inactive (C) states with the y-axis in log scale.

6 Activity periods above and below the active state threshold (separated by a red horizontal line on 5-s scale in Fig.

7 2C) were defined as active and inactive periods, respectively. (D-G) Mean and standard deviations of NPD of 8 residence times in active state (red) and in inactive state (blue) among individual animals in indicated time regime 
9 were shown in log-log plot. Fitting was performed over $0.3-5.0 \mathrm{~s}$ for active states and over $1-50 \mathrm{~s}$ for inactive

0 states. The fit line is shown as a black line. Pre-starved vs. Starved time regimes are as described in Figure 2

1 (wild-type) and the main text (egl-4 mutants).

\section{Figure 4 Multifractal analysis of numerically-generated round series and experimentally}

4 obtained round series of active and inactive states in a representative $C$. elegans animal

5 (A-D) MF-DFA of numerically-generated round series with monofractal-like (A) and multifractal (B) properties.

6 Time series with small/middle/great multifractality were generated by the multiplicative cascading processes

7 using log-normal functions whose variances were determined by random noises with small/middle/large variances

8 (41,42; see Supplemental Information). Experimentally-obtained Pre-starved active/inactive state round series of

9 High activity wild-type (C) and egl-4 mutant (D) animals. Time series [blue, in (A) and (B), magnified by arbitrary

0 unit] and cumulative sum of the deviations from the average of values in the time series [red, in (A), (C), and (D)]

1 are shown in upper three (A and B) and two (C and D) graphs. $F(q, s)$ vs. scale $s$ plot data (dots) and their fit

2 functions (lines) are shown at lower $q$ values (cold colors) and higher $q$ values (hotter colors) in a range of $-10<$

$3 \mathrm{q}<10$ [left column in the lower three (A and B) and two (C and D) graphs]; corresponding multifractal spectra

4 are shown in right columns of each graph. Original white, pink, and Brown noise time series are shown, 5 respectively, after $10,10^{2}$, and $10^{4}$-time magnifications (A).

\section{$7 \quad$ Figure 5 Multifractal spectra averaged from multiple animals}

8 Multifractal spectra means (and standard deviations) were calculated from round series data spectra of active 9 (left) or inactive (right) states from multiple wild-type animals in High (A), Middle (B), and Low (C) activity 0 classes in the Pre-starved (Pre-S, red circles) or Starved (S, blue circles) time regimes. For egl-4 mutants (D), 1 spectra represent only in Pre-S (red circles) time regime. Note that the spectrum width in Pre-starved time regime 
2 was as narrow as in Starved time regime in Low activity classes, which was due to too short high activity period

3 during the defined Pre-starved regime among animals in Low activity classes (C).

\section{$5 \quad$ Figure 6 Multifractal episodic swimming in C. elegans.}

6 (A) Alternate state transitions are driven by distinct kinetics: active-to-inactive and inactive-to-active state

7 transitions follow narrow and wide multifractal kinetics, respectively. (B) Because C. elegans episodic swimming

8 is driven by alternating transitions between an actively moving state and an inactive (resting and posing) state

9 over a broad range of temporal scales (i.e. seconds to hours) the residence-time round series data of which are

0 characterized by a multifractal nature, we refer to this behavior as "multifractal episodic swimming". (C)

1 Summary of our kinetic analyses. See Tables 1 and 2.

2

3 
(A)

bioRxiv preprint doi: https://doi.org/10.1101/2020.04.22.056606; this version posted April 24, 2020. The copyright holder for this preprint (which was not certified by peer review) is the author/funder, who has granted bioRxiv a license to display the preprint in perpetuity. It is made
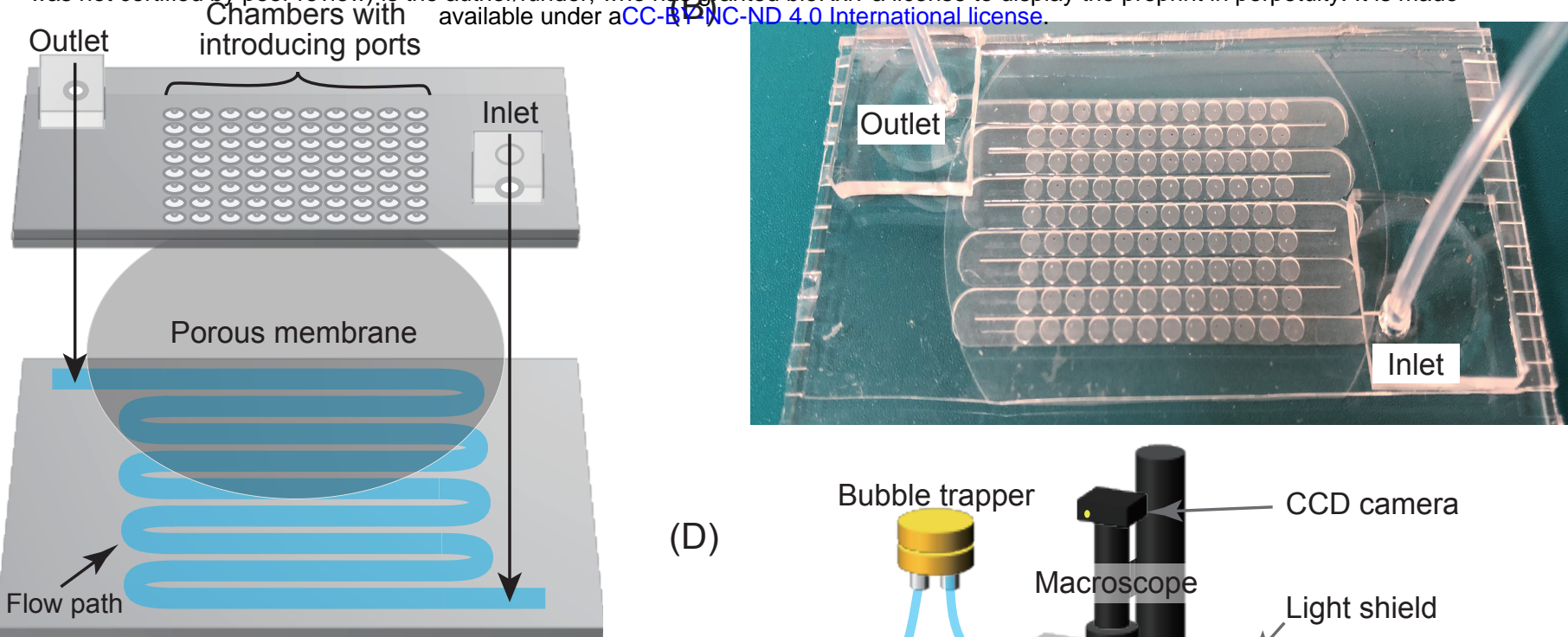

(C)

\section{Introducing port}

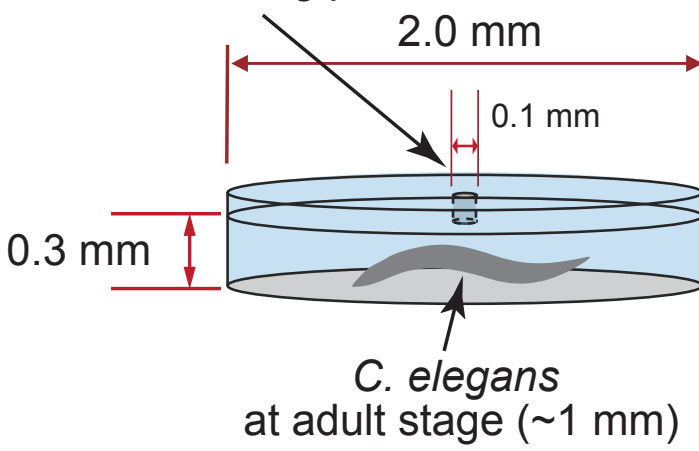

(D)

\section{Bubble trapper}
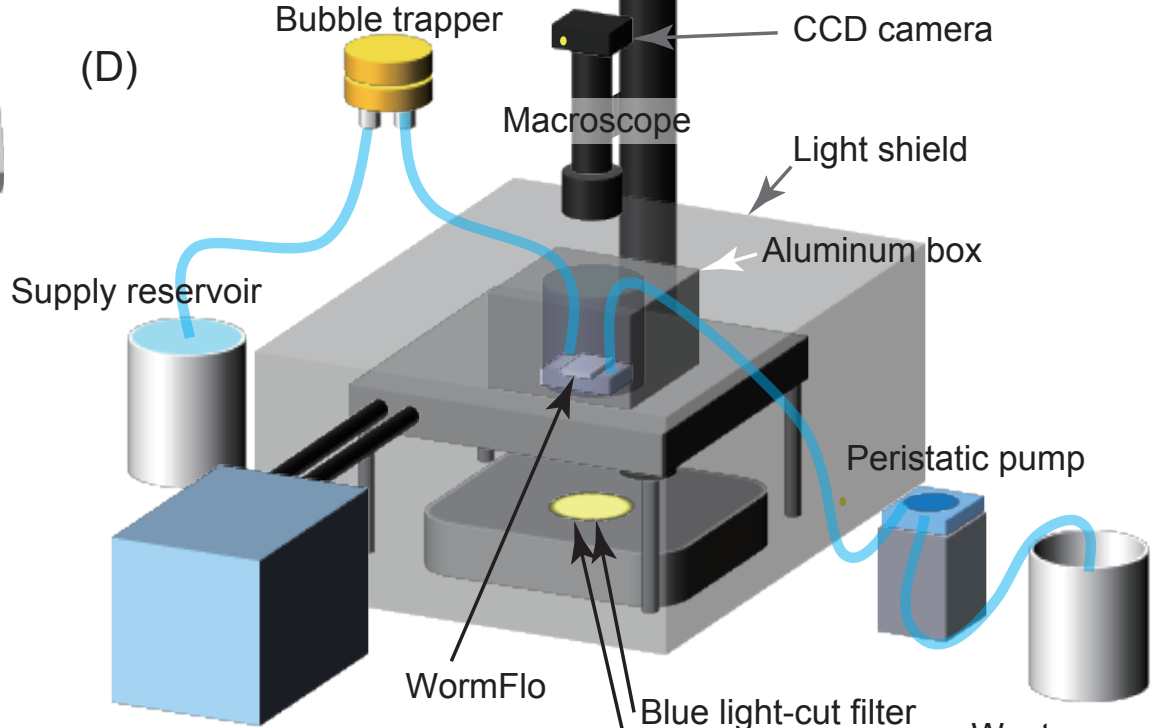

Temperature-controlling water bath

Waste reservoir

Figure 1_Ikeda et al 
bioRxiv preprint doi: https://doi.org/10.1101/2020.04.22.056606; this version posted April 24, 2020. The copyright holder for this preprint (which

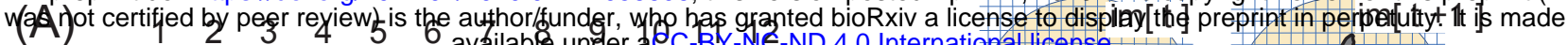

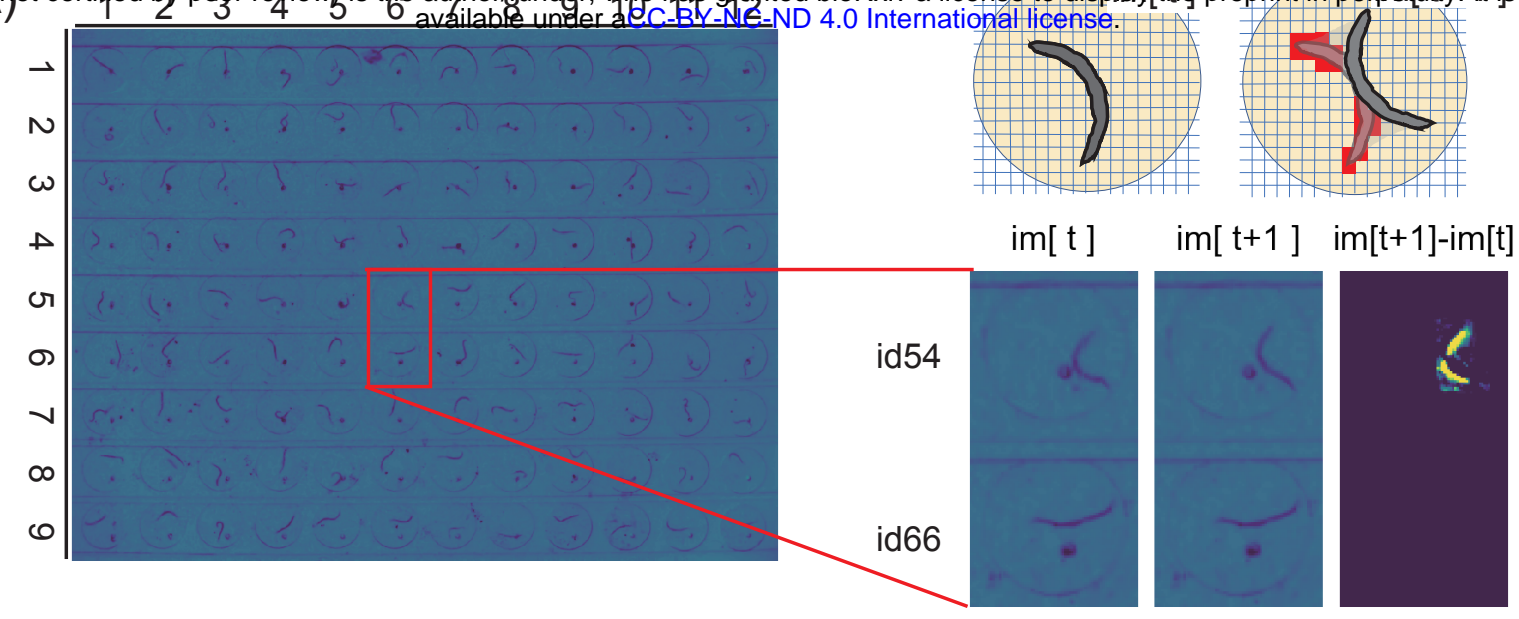

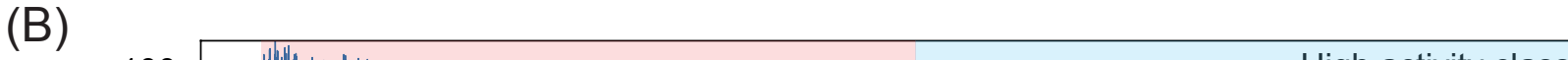

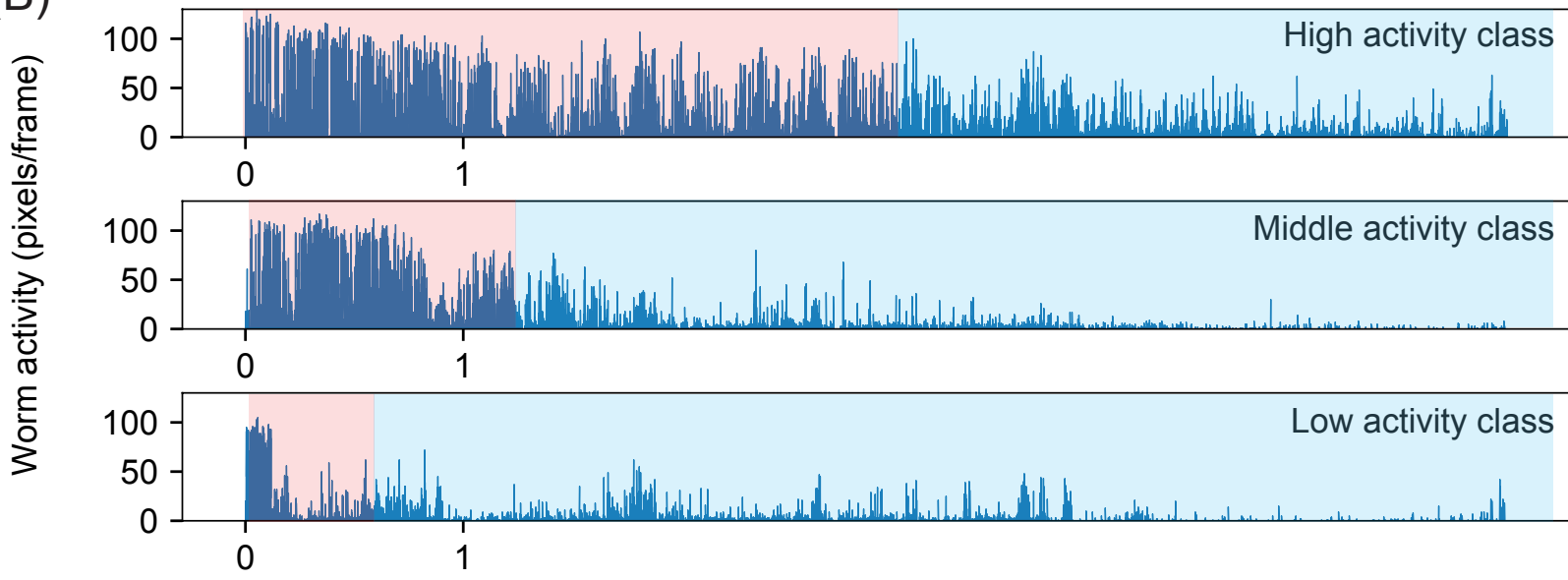

[day]

[day]

[day]

(C) $\begin{array}{r}100 \\ 50- \\ 0\end{array}$
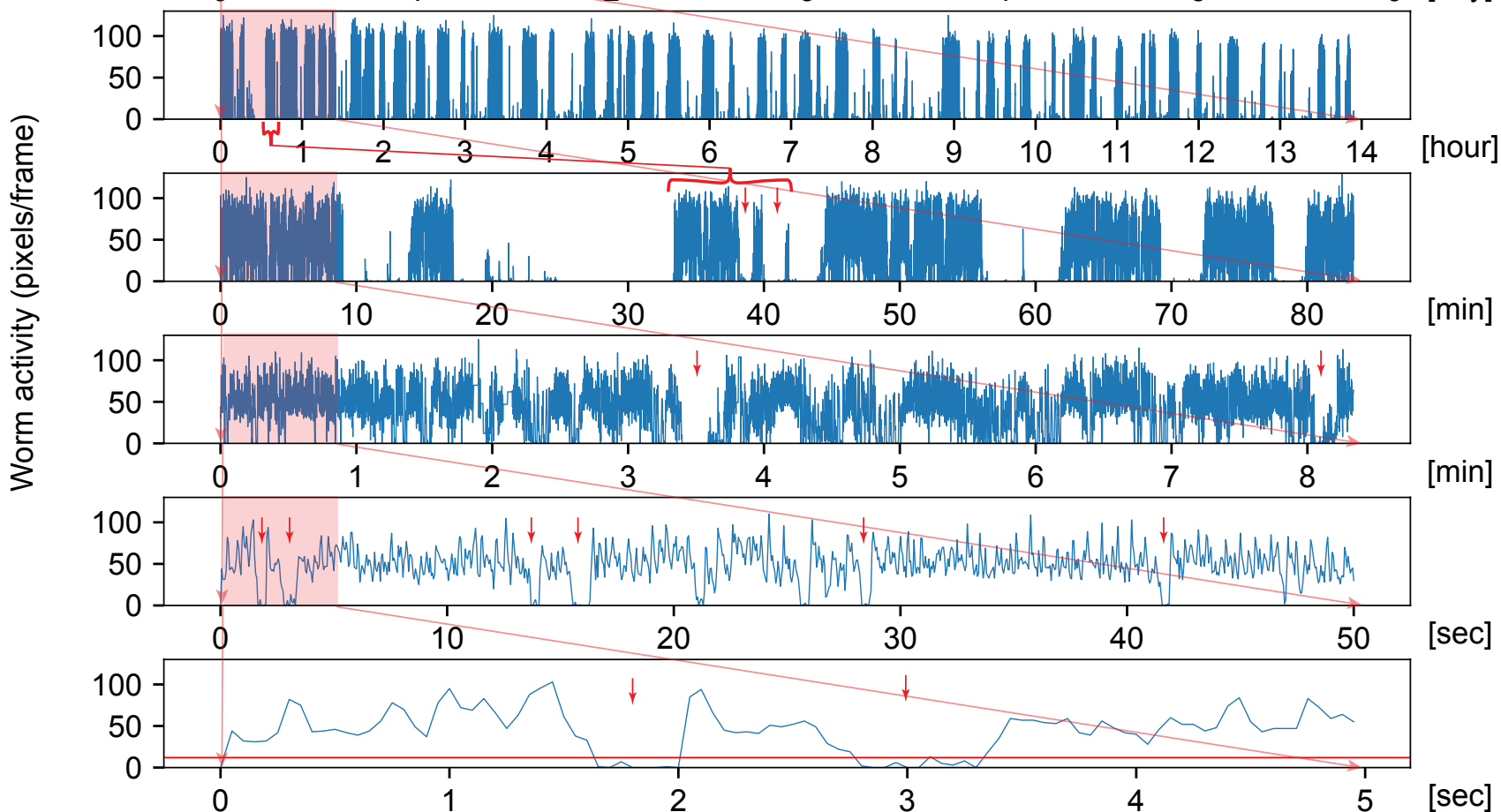

Figure 2_Ikeda et al 
(A) Priv preprint doi: https://doi.org/10.1101/20B3.04.22.056606; this version posted April 24, 2020. The copyright holder for this preprint (which
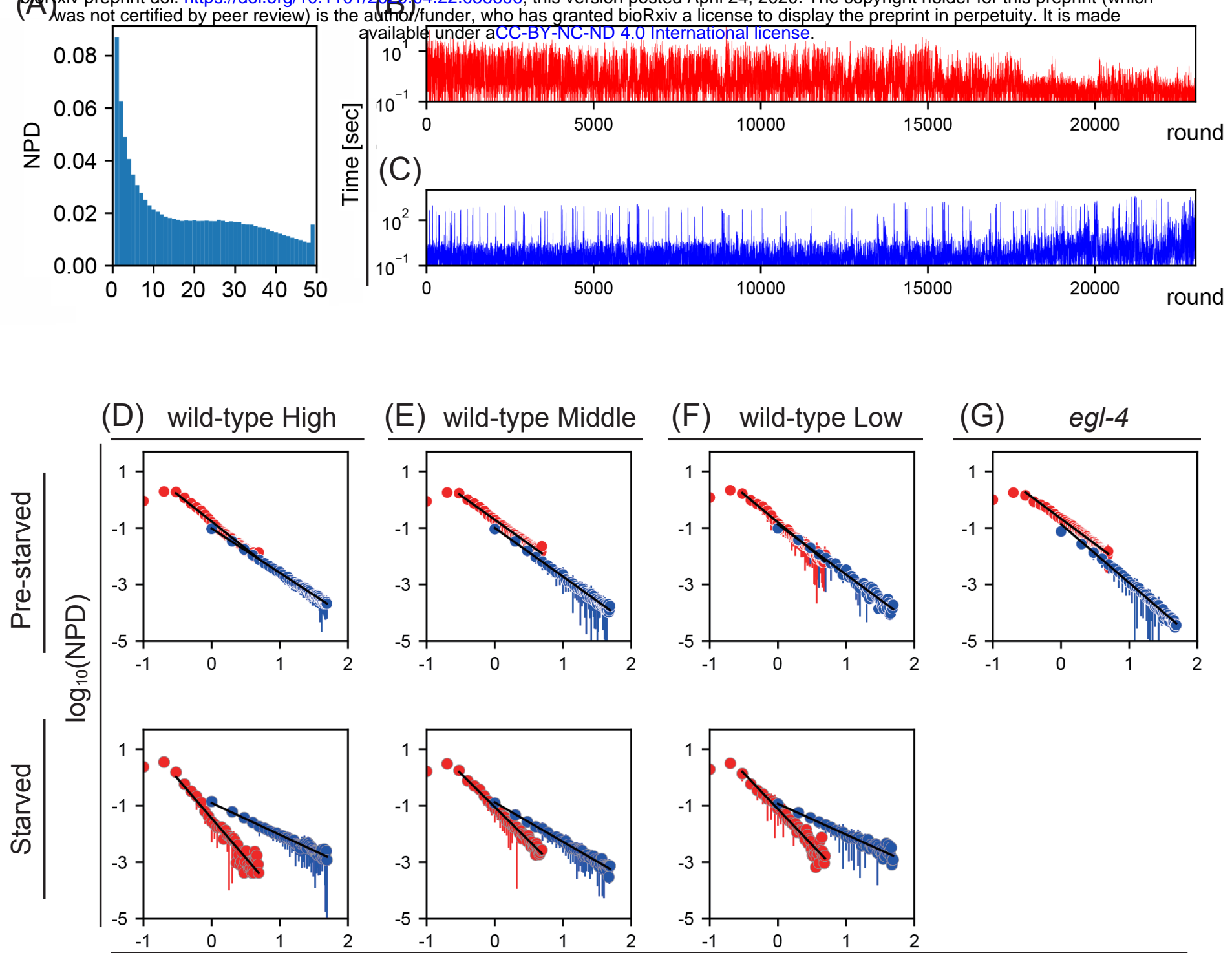

(E) wild-type Middle
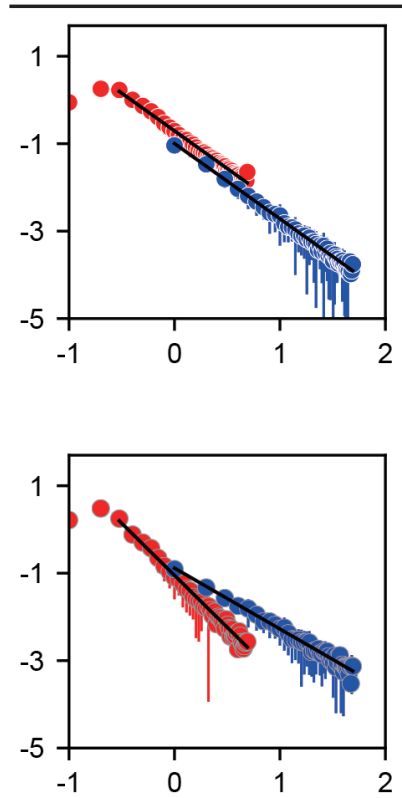

(F) wild-type Low

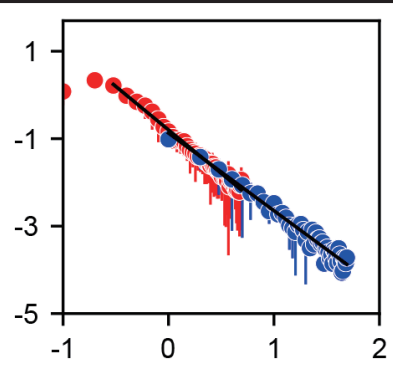

(G) egl-4

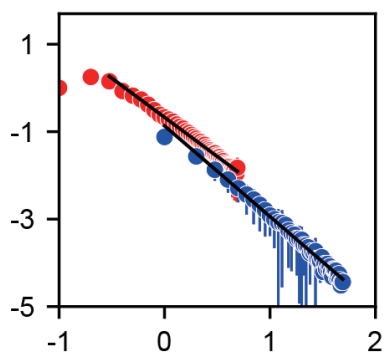

Figure 3 Ikeda et al 


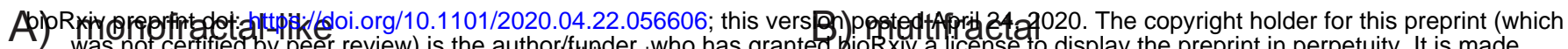
was not certified by peer review) is the author(fwhideroisho has granted bioRxiv a license to display the preprint in perpetuity. It is made available under aCC-BY-NC-ND 4.0 Interq Đitonal license
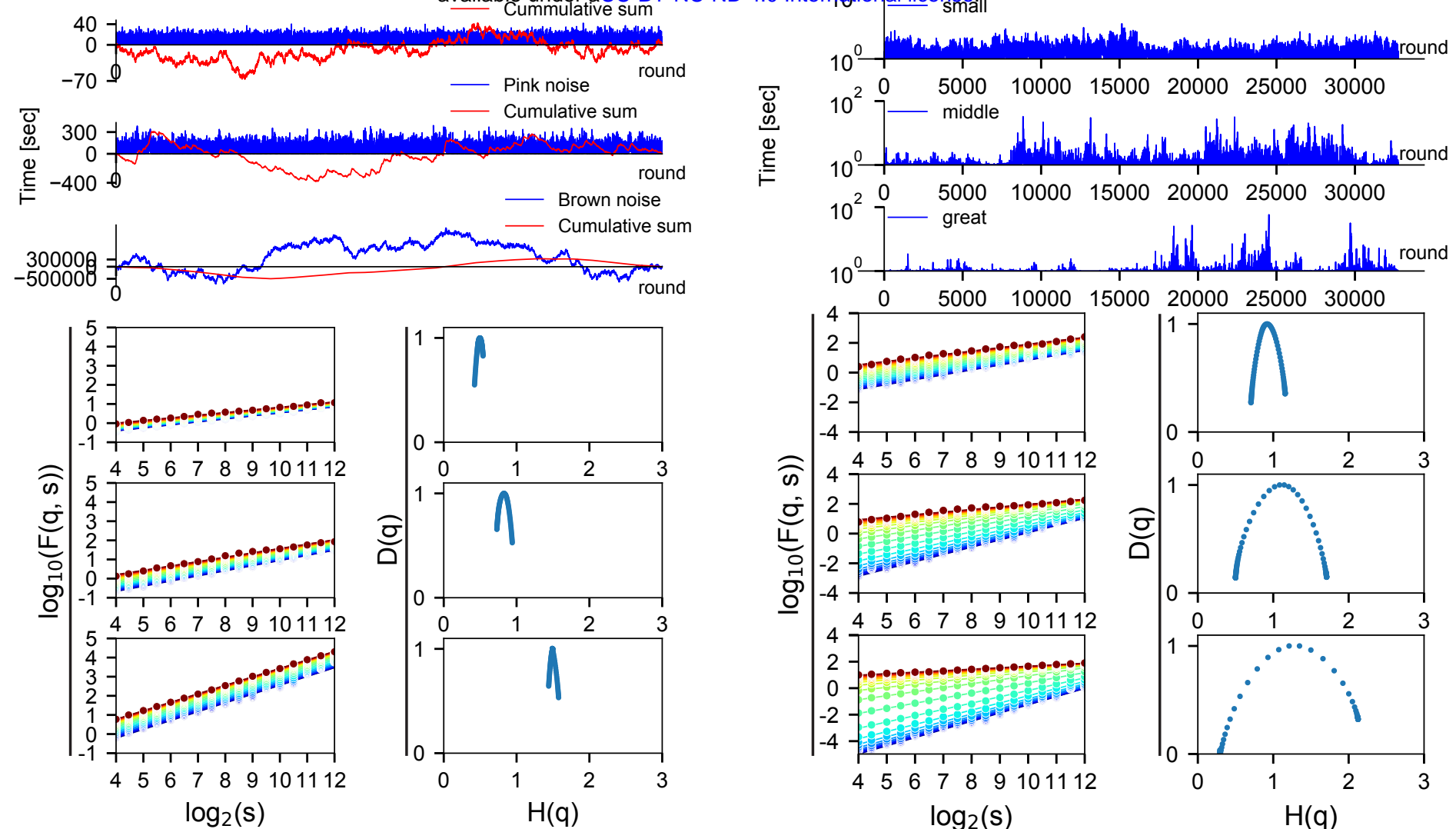

C) wild-type High Pre-starve

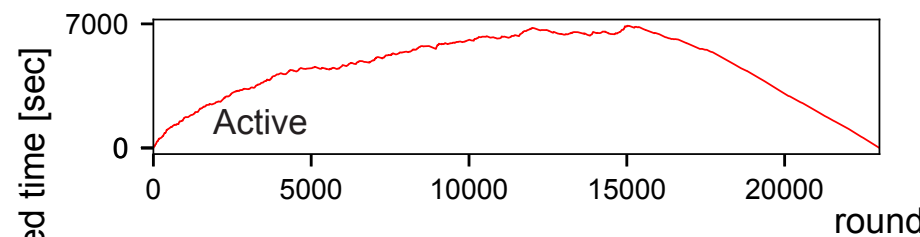

D) egl-4 Pre-starve

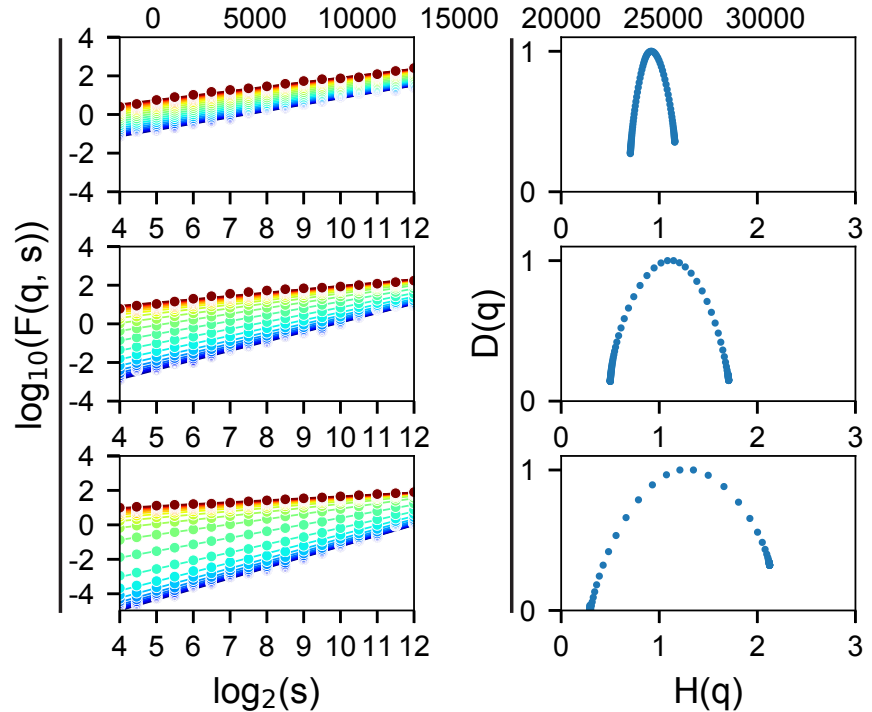

$\begin{array}{lllllll}4 & 5 & 6 & 7 & 8 & 9 & 1011\end{array}$

$\log _{2}(s)$
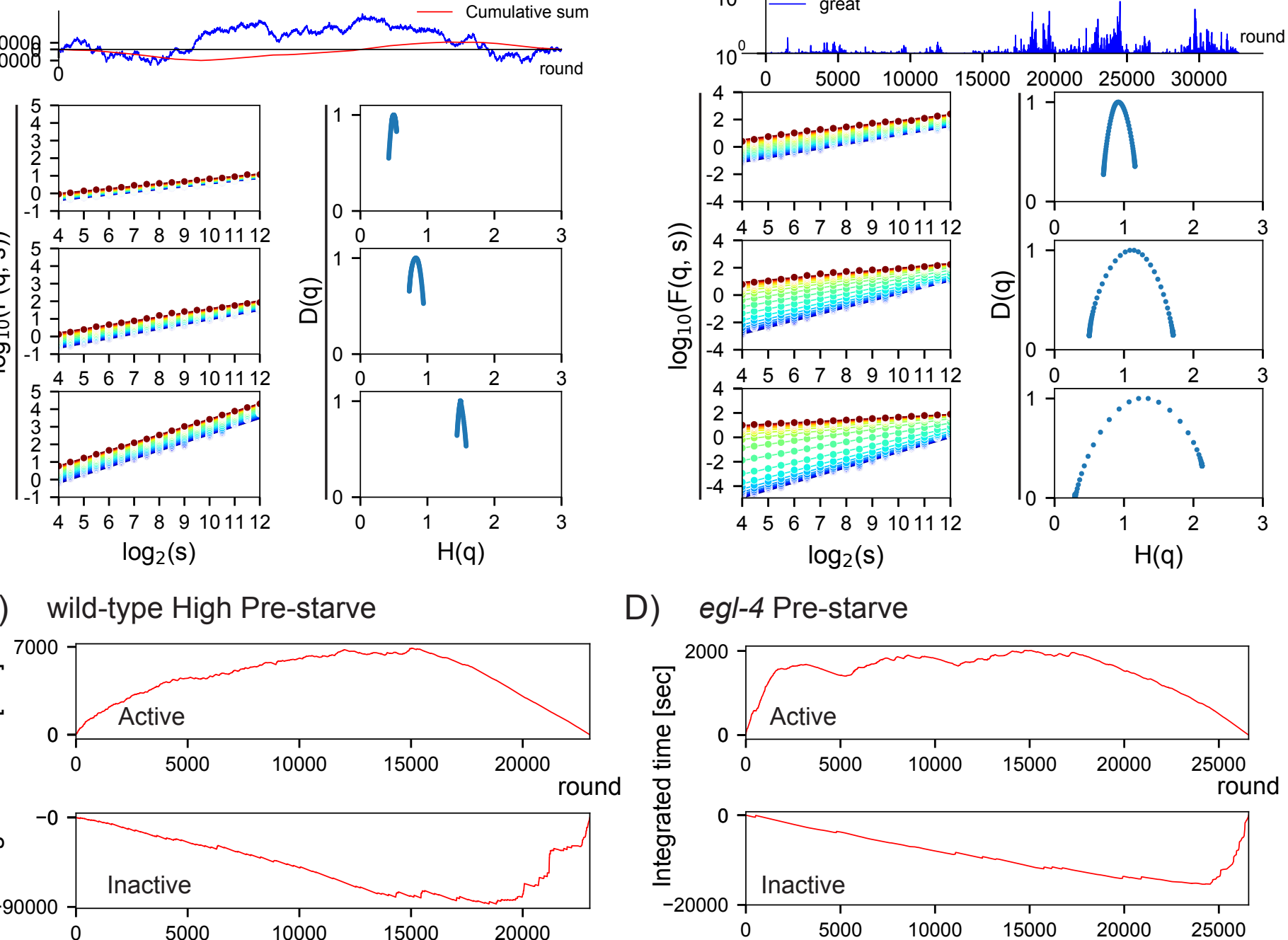

round
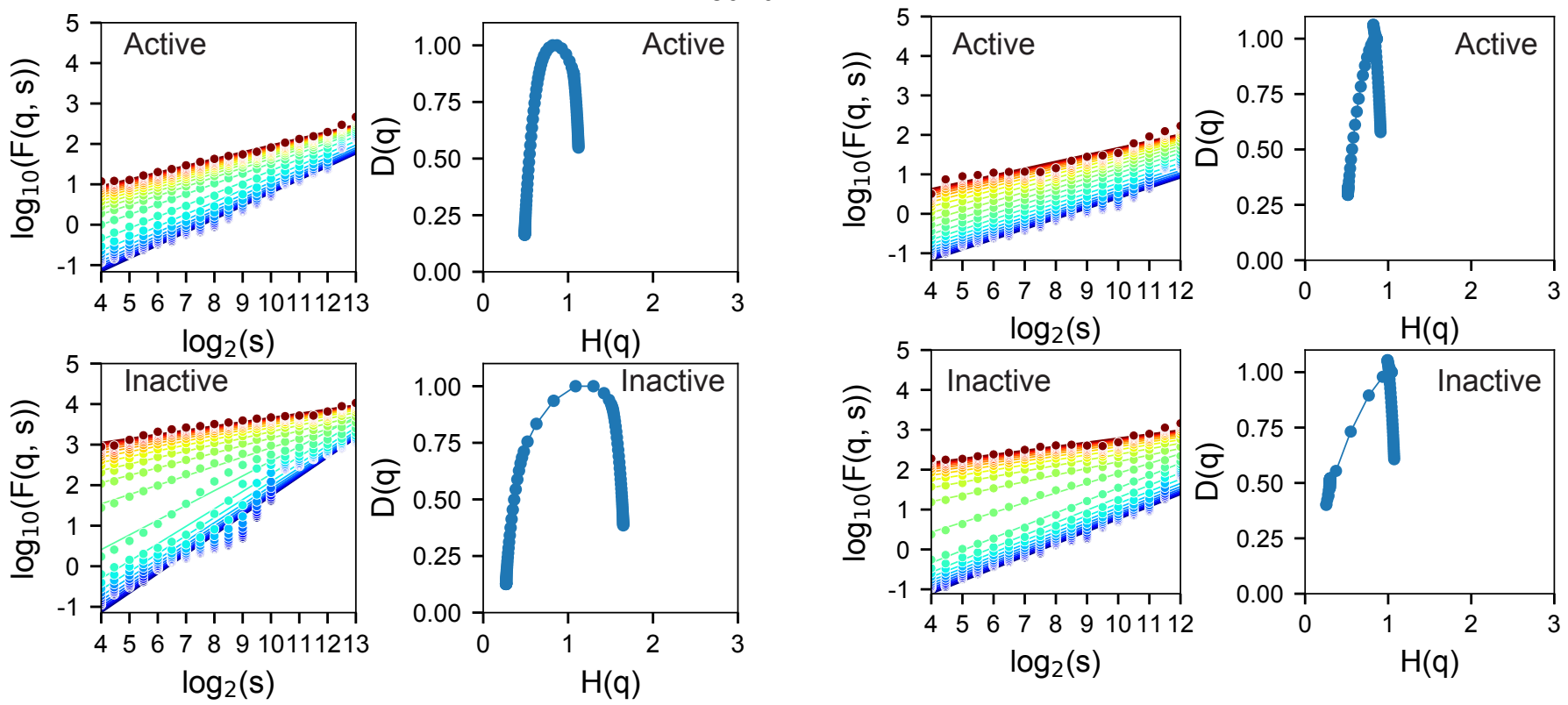

Figure 4 Ikeda et al 
bioRxiv preprint doi: https://doi.org/10.1101/2020.04.22.056606; this version posted April 24, 2020. The copyright holder for this preprint (which was not certified by peer review) is the author/funder, who has granted bioRxiv a license to display the preprint in perpetuity. It is made available under aCC-BY-NC-ND 4.0 International license.

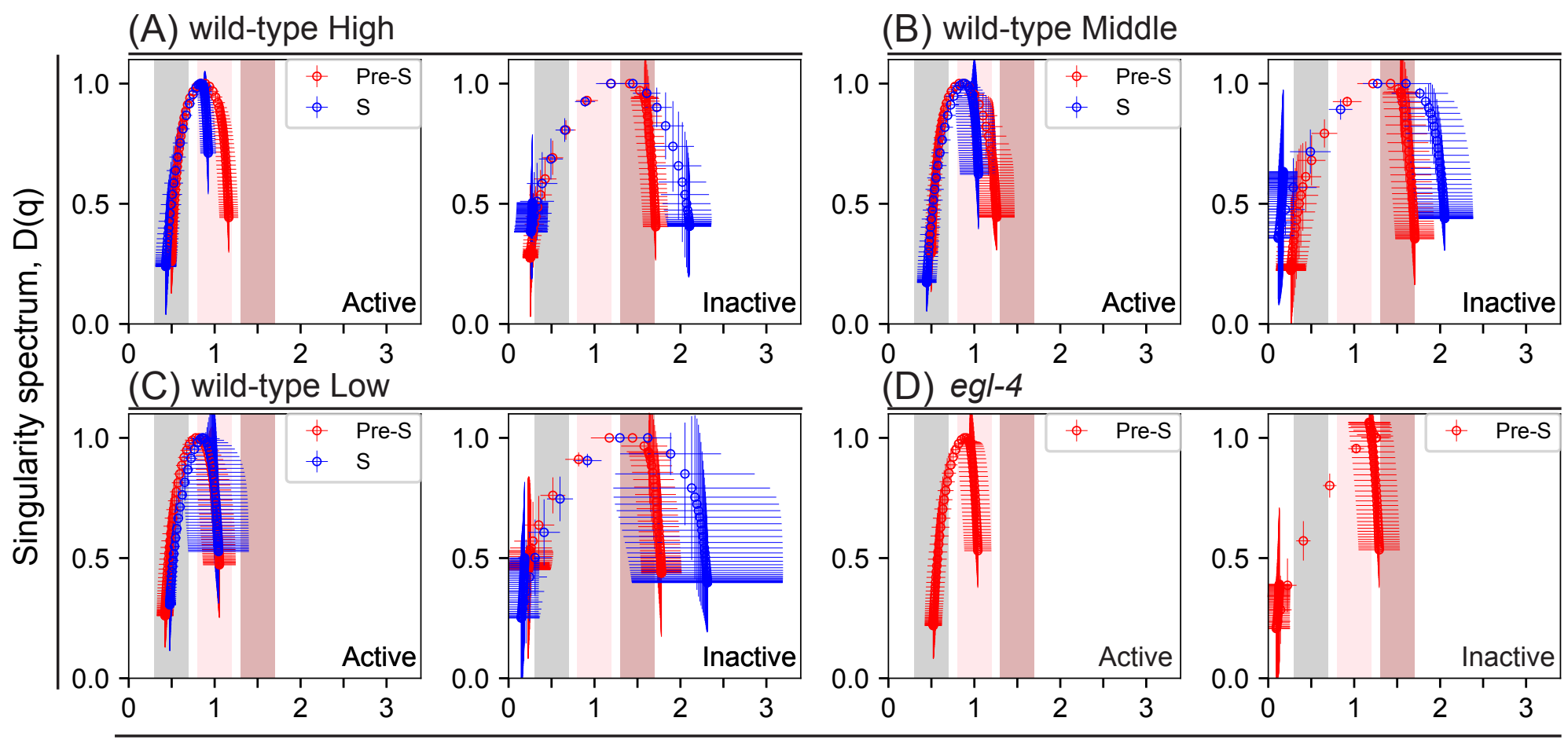

Hölder spectrum, $\mathrm{H}(\mathrm{q})$ 
bioRxiv preprint doi: https://doi.org/10.1101/2020.04.22.056606; this version posted April 24, 2020. The copyright holder for this preprint (which was not certified by peer review) is the author/funder, who has granted bioRxiv a license to display the preprint in perpetuity. It is made

(A) available under aCC-BY-NC-ND4.P International license

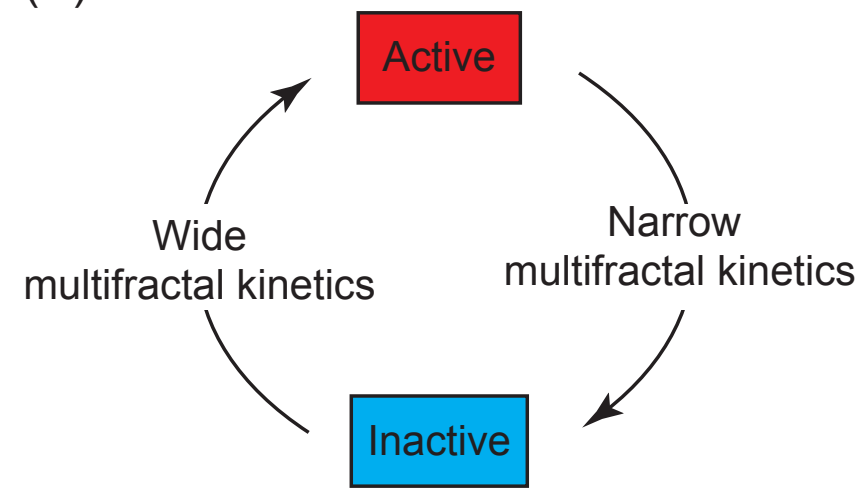

(B) International license Multiractal episodic swimming

Wide

Narrow

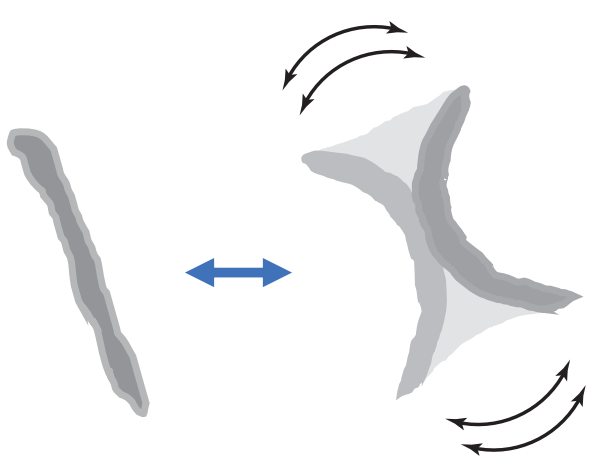

Time scale ranging from second to 1 -hour

(C)

\begin{tabular}{ccccccc} 
Variables & Indicator for & $\begin{array}{c}\text { Molecular } \\
\text { mechanism for }\end{array}$ & Behavioral states & \multicolumn{2}{c}{ Wild-type } & egl-4 mutants \\
$\begin{array}{l}\text { Power law } \\
\text { exponent }\end{array}$ & $\begin{array}{c}\text { Appearance frequency } \\
\text { of residence time }\end{array}$ & $\begin{array}{c}\text { Scale-free } \\
\text { residence time }\end{array}$ & Active & - & Starved & Pre-starved \\
\hline \multirow{2}{*}{$H_{\text {peak }}$} & $\begin{array}{l}\text { Memory in } \\
\text { residence time }\end{array}$ & $\begin{array}{c}\text { Behavioral } \\
\text { memory }\end{array}$ & $\begin{array}{c}\text { Active } \\
\text { Inactive }\end{array}$ & - & shallow & steep \\
Width & $\begin{array}{l}\text { Variety of } \\
\text { cluster structure }\end{array}$ & $\begin{array}{c}\text { Behavioral } \\
\text { complexity }\end{array}$ & Active & - & - & - \\
Inactive & - & short \\
\hline
\end{tabular}

Figure 6_ Ikeda et al 